Revue des patrimoines

\title{
Les gardians de Camargue et leurs chevaux, patrimoine archivistique et photographique pour une étude historique
}

\section{Frédéric Raynaud}

\section{OpenEdition}

Journals

Édition électronique

URL : http://journals.openedition.org/insitu/12105

DOI : 10.4000/insitu. 12105

ISSN : 1630-7305

Éditeur

Ministère de la Culture

Référence électronique

Frédéric Raynaud, «Les gardians de Camargue et leurs chevaux, patrimoine archivistique et photographique pour une étude historique », In Situ [En ligne], 27 | 2015, mis en ligne le 02 novembre 2015, consulté le 14 novembre 2019. URL : http://journals.openedition.org/insitu/12105 ; DOI : 10.4000/insitu. 12105

Ce document a été généré automatiquement le 14 novembre 2019.

\section{(c) (i) $(9)$}

In Situ Revues des patrimoines est mis à disposition selon les termes de la licence Creative Commons Attribution - Pas d'Utilisation Commerciale - Pas de Modification 4.0 International. 


\title{
Les gardians de Camargue et leurs chevaux, patrimoine archivistique et photographique pour une étude historique
}

\author{
Frédéric Raynaud
}

\author{
À la mémoire d'Hubert Yonnet (1926-2014), \\ président de l'Antico counfrarié di gardian de \\ bouvino e roussatino \\ À la mémoire de Jean Mathieu (1948-2014), \\ président de l'Association des gardians \\ professionnels
}

1 Avant les travaux d'endiguement réalisés au milieu du XIX ${ }^{e}$ siècle, les caractères géographiques du delta du Rhône faisaient que de grands espaces, soumis aux remontées salines, n'étaient exploitables que par l'élevage extensif de bovins et de chevaux. La gestion de ces troupeaux était assurée par un pasteur à cheval désigné par le terme provençal de gardian, désignant un ouvrier agricole employé dans les grandes exploitations qu'étaient les mas de Camargue depuis le début de l'époque moderne. Dans son dictionnaire des langues d'oc, Frédéric Mistral présente cependant une seconde définition correspondant à: "celui qui mène au pâturage commun, les chevaux, mulets et vaches d'un village »; ce cas n'est cependant pas encore documenté et pourrait concerner plus particulièrement la Petite Camargue, soit la rive droite du Rhône, espace quasiment désert jusqu'à la fin du XIX siècle et où de vastes marais dépendaient des grandes abbayes ou de domaines seigneuriaux mais également communaux. L'histoire du métier de gardian peut être retracée depuis le Moyen-Âge, dans le cadre du territoire relevant de la ville d'Arles, appréhendé grâce à son importante documentation historique dont une synthèse générale a été publiée sous la direction de Jean-Maurice Rouquette et dont Louis Stouff a plus particulièrement étudié la période médiévale et Damien Carraz a analysé les domaines des Templiers. La profession de gardian est plus spécifiquement connue à partir de l'étude des deux 
registres de la confrérie de Saint-Georges, conservés pour la période de l'Ancien Régime $^{1}$. Des récits de voyageurs et la synthèse des rapports d'officiers des haras réalisée par le vétérinaire Christian Berriot fournissent des renseignements sur la période allant du XVII ${ }^{e}$ au XIX ${ }^{e}$ siècle. Ces informations sont complétées, pour l'époque contemporaine, par des travaux d'ethnographes et de zoologues. Elles sont spécifiquement illustrées par les travaux des photographes Gaston Bouzanquet (1866-1937) et Carle Naudot (1880-1948), réalisés au début du xxe siècle dont les collections ont été déposées au musée de la Camargue, à Arles.

2 À partir du début du XIX siècle et à l'occasion des fêtes patronales ou nationales, les municipalités organisèrent le programme des réjouissances autour de jeux taurins. L'image du gardian connut ainsi une évolution : de simple ouvrier agricole, solitaire et un peu sauvage, sous l'Ancien Régime, il devint l'acteur indispensable des fêtes populaires. Depuis la fin du XIX siècle, avec la renaissance culturelle provençale, représentée en Camargue par deux illustres félibres ${ }^{2}$ issus de classes sociales privilégiées mais ayant choisi le dur métier d'éleveur, par passion, le marquis Folco de Baroncelli-Javon (1869-1943) et l'écrivain Joseph d'Arbaud (1874-1950), le gardian incarne le preux chevalier, défenseur des traditions et d'un art de vivre ancestral. Le sujet de prédilection reste le taureau de Camargue et les jeux collectifs auxquels il participe et le vif intérêt de la population des deux sexes pour le monde de la «bouvine » incite de nombreuses personnes à participer bénévolement aux travaux des éleveurs. Après les périodes des années 1950-1970, sous l'influence de la mode western, et à partir des années 1980, de l'équitation ibérique, l'engouement actuel pour les pratiques équestres a entrainé l'utilisation du cheval camargue dans toutes les disciplines de l'art équestre et l'apparition d'une équitation de loisirs spécifique au cheval de Camargue dite monte gardianne, dont l'apprentissage se déroule généralement en carrière et qui donne lieu à des compétitions sportives issues des spécificités du travail. Traditionnellement, le gardian expérimenté ne disait rien, il fallait simplement l'imiter, savoir se placer dans le groupe et se rendre utile et ainsi se pratiquait une sélection rigoureuse parmi les élèves motivés. Bien que contraint de s'adapter à la parcellisation actuelle du territoire et à l'enclosure des espaces de pâturage, le savoir-faire spécifique du métier ne s'en transmet pas moins suivant des pratiques éprouvées, réputées ancestrales, avec son vocabulaire provençal, tout en sachant s'adapter à l'évolution zootechnique, ce qui est particulièrement le cas pour la conduite des poulains beaucoup plus imprégnés et manipulés dès leur naissance et ainsi beaucoup plus faciles à éduquer.

\section{Caractères et gestion agraire du territoire camarguais}

3 Port important au carrefour des voies maritimes, fluviale et terrestres durant l'Antiquité, préfecture des Gaules au v viècle, siège d'archevêché dès le IV siècle, capitale du royaume de Bourgogne, siège du pouvoir comtal et occupant une position frontalière avec le royaume de France jusqu'à ce que Raimond Bérenger V installe le Parlement à Aix en 1250, la cité d'Arles disposait d'un vaste territoire s'étendant sur 110000 hectares. Cet espace se répartit en quatre quartiers : plaine alluviale en amont, le Trébon, vaste steppe caillouteuse d'un ancien lit de la Durance, la Crau, l'île de Camargue, entre les deux bras principaux du Rhône où l'espace du delta est partagé avec la commune des Saintes-Maries-de-la-Mer et la rive gauche du Grand Rhône, le 
Plan de bourg. C'est un terroir diversifié aux abondantes ressources agricoles variées ayant permis la constitution d'importants domaines. Le delta présente dans sa partie nord, une formation fluviatile de riches apports alluviaux déposés par les ramifications du Rhône, entrecoupée de marais et d'étangs et au sud, un espace de dépôts marins et éoliens marqué par une forte salinité des sols, les rendant totalement impropres à l'agriculture. Ne présentant que des surfaces horizontales et culminant à $5 \mathrm{~m}$ d'altitude maximum, le pays est soumis à tous les vents, certains comme le mistral ou les vents d'est pouvant être particulièrement violents et glacials en hiver mais ce terroir connaît fréquemment des étés torrides desséchant les pâturages. Milieu naturel préservé, les marais, cordons littoraux et dunes offrent une végétation variée, mais plus ou moins nutritive et peu abondante nécessitant une surface assez importante par tête de bétail. Dans les marais poussent le cypérus et surtout le roseau phragmite dont la valeur nutritive est proche de celle de la luzerne, apprécié des chevaux et qui était récolté en été pour fournir un fourrage distribué en hiver. Sur les bords et les parties émergées croissent tirasse, une sorte de chiendent, soude, salicorne et saladelle alors que sur les terres plus élevées, les graminées et composées fournissent une riche nourriture précoce mais qui disparaît rapidement avec la sécheresse.

4 Du fait de son caractère peu hospitalier, la Camargue n'a jamais été densément peuplée et l'espace était partagé entre de grandes propriétés. Au Moyen-Âge, la plus grande partie appartenait à des ecclésiastiques : l'archevêque, le chapitre cathédral, le couvent Saint-Césaire d'Arles et des abbayes bénédictines et cisterciennes, plus particulièrement en Petite Camargue : Saint-Gilles, Psalmodi et Franquevaux. À partir du début du XII ${ }^{\mathrm{e}}$ siècle, les ordres militaires : templiers et hospitaliers de Saint-Jean-deJérusalem et même, de manière éphémère, les chevaliers teutoniques, furent dotés de grands domaines camarguais et menèrent une politique d'extension territoriale qui dut être limitée par les autorités au XIII ${ }^{\mathrm{e}}$ siècle. Outre pour leur propre usage, l'archevêque comme les abbés trouvaient un intérêt particulier dans l'élevage des chevaux, offerts en cadeau diplomatique aux puissants dont ils voulaient s'attirer la bienveillance ou donnés à des vassaux dont ils voulaient s'attacher les services. Judicieusement implantés à proximité des ports de Marseille ou d'Aigues-Mortes, les frères des ordres militaires de la basse vallée du Rhône étaient chargés du ravitaillement et de la remonte des armées croisées : on peut imaginer qu'ils donnèrent un élan particulier à l'élevage des chevaux dans la région, étant donné le besoin permanent des chevaux de remonte pour les armées croisées. Le reste du territoire relevait des domaines du comte et de grands féodaux, comme les seigneurs des Baux ou de chevaliers urbains, catégorie sociale attachée à la possession de montures de qualité. Bénéficiant d'un niveau de fortune les mettant sur un pied d'égalité avec la noblesse avec laquelle ils partageaient le pouvoir municipal, les marchands d'Arles portèrent un vif intérêt à l'acquisition de propriétés en Camargue et à leur développement.

5 Jusqu'au milieu du XIve siècle, la population rurale était essentiellement regroupée dans des hameaux dispersés. Les domaines de l'archevêque et des nobles s'organisaient autour de tours, à la fois centres d'exploitation agricole et postes de défense alors que ceux des frères hospitaliers étaient gérés grâce aux habitants des " granges ». Après la crise du peuplement consécutive à la Peste noire de 1348 et des malheurs de la guerre de Cent Ans, une reprise démographique s'amorça à partir de 1430-1440 et marqua le début d'une nouvelle organisation agraire du territoire. À côté d'anciens grands domaines subsistants apparaissent des « mas » avec leur domaine propre, les afars, dont 
les principales ressources étaient la production de blé et l'élevage des ovins, correspondant à un ensemble de terres labourables et de pâturages fréquemment d'un seul tenant et groupé autour d'un habitat, le mas, ou souvent d'une cabane : au début $\mathrm{du} \mathrm{Xv}^{\mathrm{e}}$ siècle, sur 109 afars recensés dans le livre terrier, 85 sont dépourvus de mas. En évoluant dans le temps, le mas regroupa le logement du pelot, le régisseur, celui des ouvriers agricoles saisonniers ou installés, les bâtiments liés aux différentes ressources agricoles mais également un logement de plaisance pour le propriétaire et sa famille. $\mathrm{Au} \mathrm{XVI}{ }^{\mathrm{e}}$ siècle, le nombre de mas s'accroît. Les hospitaliers ont pratiquement abandonné l'exploitation directe de leurs domaines, l'Église, les nobles et les bourgeois octroient des baux favorables à des laboureurs pour des espaces compris entre 30 et 200 ha de terres et de pâturages, comportant occasionnellement des marais et des bois. Lors de la vente des biens nationaux, à partir de 1792, les grands mas de Camargue furent achetés par des notables d'Aix, de Marseille, de Nîmes et même de Paris.

\section{Les gardians ou gardian de bouvino e roussatino}

6 C'est dans le cadre de certains de ces grand domaines particulièrement isolés et fonctionnant quasiment en autarcie que le gardian était engagé pour la garde des troupeaux de taureaux et de chevaux, désignés par le terme de manade et qui occupaient essentiellement les milieux naturels car les meilleurs pâturages étaient réservés pour les moutons. Il succède au custos vaccarum, gardien des vaches et au custos equorum, gardien des chevaux, mentionnés en 1341 parmi la domesticité au service de l'archevêché ainsi qu'aux écuyers et aux bouviers employés dans les membres des commanderies hospitalières. Dès le $\mathrm{xvI}^{\mathrm{e}}$ siècle, le métier peut se caractériser par une spécialisation : bouvier ou vacher, laissant supposer qu'il devait y avoir une séparation des genres pour les bovins. D'autres pouvaient être gardians de rosses, mot emprunté à l'ancien français désignant des chevaux farouches mais d'aucuns étaient également qualifiés d'egatié, soit gardien de troupeau de juments, manado d'ego. Cependant dans les actes notariaux et d'après la rareté de ces mentions dans les listes des confrères du XVI ${ }^{\mathrm{e}}$ siècle, les deux espèces pouvaient être, le plus souvent, associées sur un même pâturage et sous la surveillance d'un seul gardian. Chevaux et bovins vivent en bonne intelligence et la cohabitation sur un même espace ne pose pas de problème particulier (fig. 1). Les chevaux savent chasser les taureaux lorsqu'ils les jugent indésirables: " devant un bon coin d'herbe convoité, le cheval baisse les oreilles en arrière et le taureau s'enlève ${ }^{3} »$. Un contrat d'embauche de 1421 passé devant notaire stipule qu'à partir de la mi-mars, le bouvier devra coucher hors du mas, avec les bêtes, alors qu'à l'époque moderne, le gardian passait pratiquement toute l'année auprès de son troupeau. Il lui était surtout demandé de bien connaître le comportement des taureaux, de recenser les naissances et de savoir débourrer les chevaux. Fort de ces compétences, le gardian restait souvent attaché à son troupeau sur une longue période. 
Figure 1

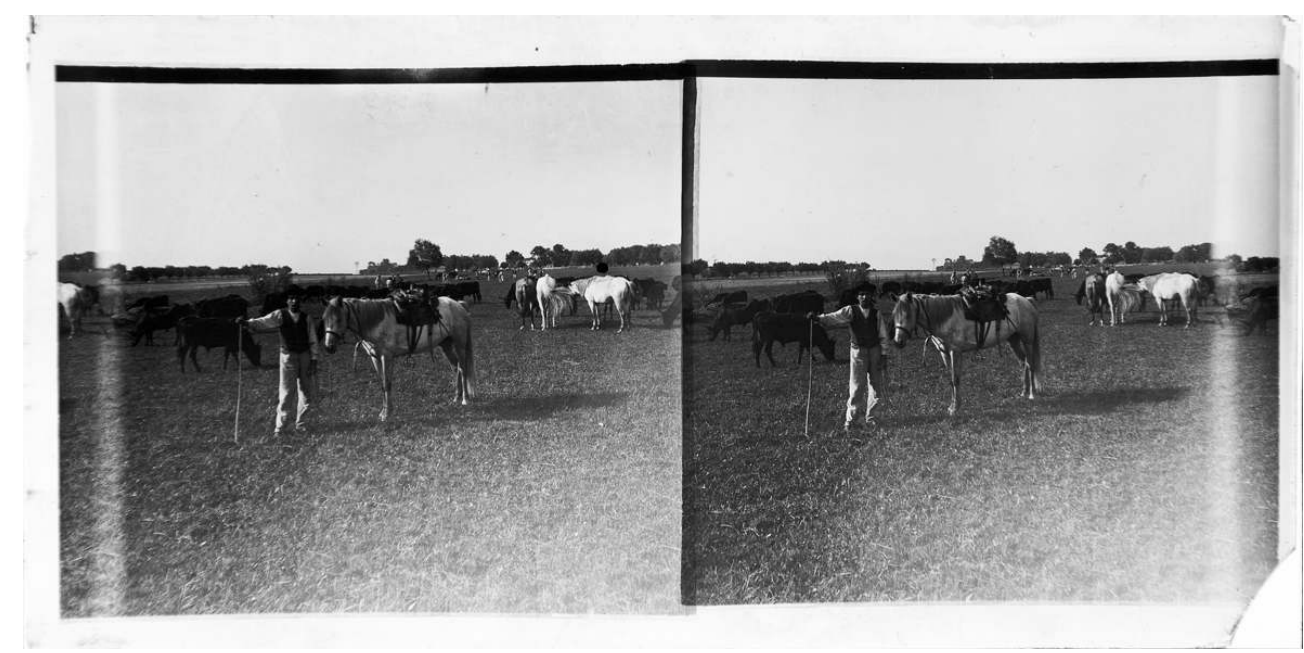

Troupeau mixte de taureaux et de chevaux entre 1910 et 1925.

Phot. Bouzanquet, Gaston, coll. Musée de la Camargue, PNR de Camargue. Num. David Huguenin (2010.01. 159). (c) Musée de la Camargue, PNR de Camargue.

7 Le gardian habitait généralement seul dans une cabane plus ou moins éloignée du mas et quand il était marié, son épouse résidait le plus souvent à Arles. Quand la cabane était proche du mas, le gardian pouvait y prendre ses repas mais rentrait toujours dormir dans sa cabane. De taille variable et de plan carré ou rectangulaire et fréquemment pourvue d'une abside au nord, la cabane est construite avec des matériaux disponibles sur place (fig. 2). Seule la façade méridionale, à laquelle est adossée la cheminée, est généralement en maçonnerie, les autres murs sont constitués de poteaux verticaux sur lesquels sont fixées des baguettes horizontales où sont liées des gerbes de roseau ou façonnés en torchis. La toiture, en sagne, variété de roseau, à deux versants, se terminant par une croupe au-dessus de l'abside, est surmontée d'un faittage de tuiles canal et recouverte de mortier de chaux sur le tiers supérieur des versants; la croupe et les bordures des pignons étaient blanchis annuellement. Le chevron axial de l'abside, débordant du toit est pourvu d'une traverse horizontale lui conférant la valeur de croix protectrice. L'espace intérieur comporte une seule pièce ou est divisé par une cloison entre pièce à vivre au sud et chambre au nord. Quelques petites fenêtres éclairent l'intérieur des plus grandes cabanes mais la porte, au sud, constitue souvent la seule ouverture des plus petites constructions. L'espace de vie se prolonge devant la façade protégé par un auvent. Ce type d'habitation n'était cependant pas spécifique aux gardians mais commun à tous les habitants de Camargue exerçant des petits métiers peu rémunérateurs. 
Figure 2

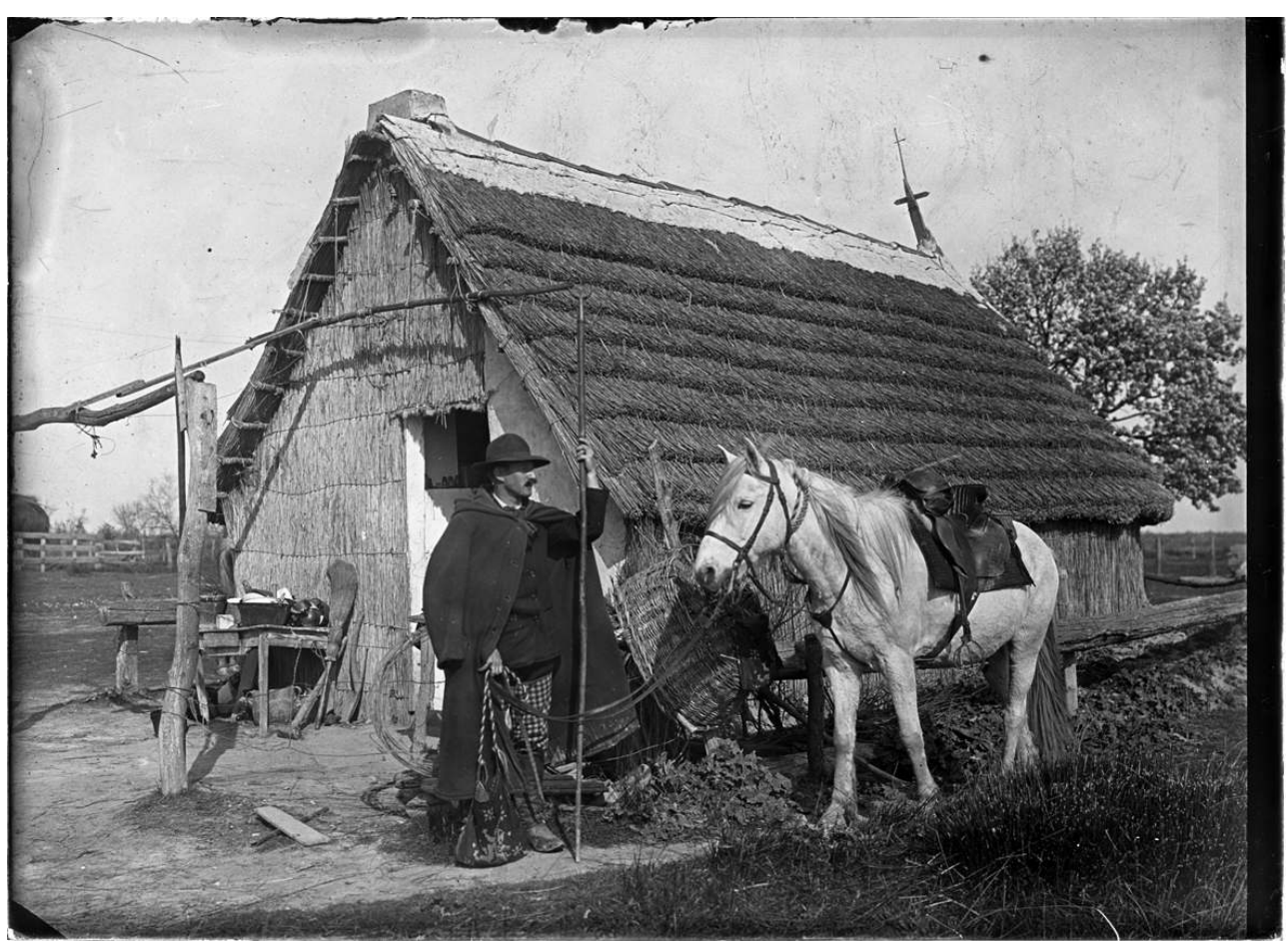

Gardian en costume d'hiver devant sa cabane entre 1910 et 1925.

Phot. Bouzanquet, Gaston, coll. Musée de la Camargue, PNR de Camargue. Num. David Huguenin (2010.01. 97). ( C Musée de la Camargue, PNR de Camargue.

\section{Le travail quotidien du gardian}

8 Consistant essentiellement à surveiller des troupeaux pouvant compter plusieurs centaines de têtes réparties sur des grands espaces, le travail quotidien du gardian était grandement facilité par l'isolement des manades dans des pâtures environnées par le Rhône, les étangs et les canaux d'irrigation qui formaient des limites naturelles, avant que la pose de clôtures ne cloisonne l'espace. De ce fait il y avait relativement peu de risques de mélanges des troupeaux ou de dégâts causés aux terres cultivées. Comme l'explique le gardian André Bouix : "suivant un parcours immuable, les bêtes partent le matin de tel endroit et y reviennent le soir et si l'une d'elles s'égare, c'est qu'elle le veut bien. La manade avance en mangeant, ce sont toujours les mêmes qui tirent comme ce sont toujours les mêmes qui sont à la traîne, ce sont donc ces animaux qui seront équipés de cloches $^{4} »$. Ces derniers sont d'une grande utilité pour la conduite des troupeaux : castrés et équipés de cloches, les simbèu ou dountaire, expérimentés car souvent utilisés sur des périodes allant de dix à vingt ans et connaissant les demandes de l'éleveur, ils servent de meneurs et d'objet de ralliement pour les taureaux échappés ou poursuivis. 
Figure 3

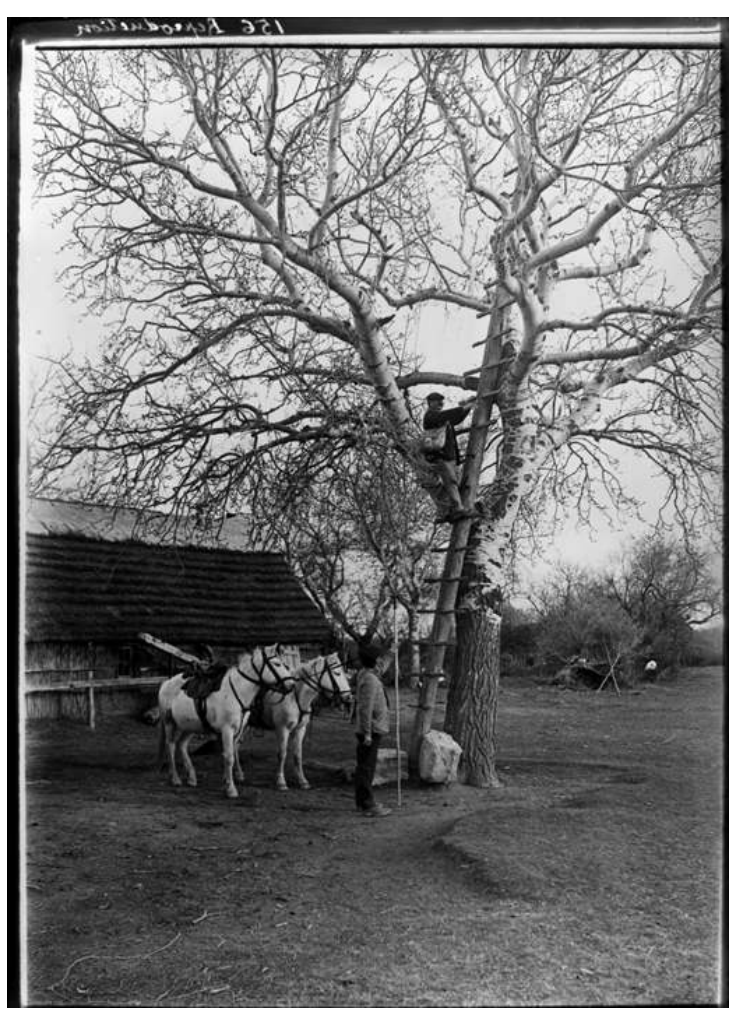

Gardian monté sur son escalassoun, procédant au repérage des troupeaux avant 1948.

Phot. Naudot, Carle, coll. Musée de la Camargue, PNR de Camargue. Num. David Huguenin (8301743). (c) Musée de la Camargue, PNR de Camargue.

9 Au matin, le gardian pouvait grimper sur un escalassoun, grand poteau pourvu de barreaux insérés latéralement, pour localiser les troupeaux (fig. 3) et les rejoindre, pourvu d'un simple bâton, le calos. La garde quotidienne se déroulait souvent à pied à bastoun planta, le gardian appuyé sur son bâton et tenant son cheval par la bride (fig. 4). Cette activité laissait suffisamment de temps pour que le gardian puisse s'occuper de son ravitaillement grâce au braconnage et à la pêche. Les lapins qui pullulaient en Camargue, accoutumés aux bruits de piétinement des troupeaux, pouvaient être adroitement assommés d'un simple coup de calos et constituaient fréquemment l'ordinaire des gardians. Apparemment, jusqu'à la fin du XIX ${ }^{\mathrm{e}}$ siècle, quelques cavaliers pouvaient organiser une battue aux perdreaux, les poursuivant jusqu'à épuisement et les poussant jusqu'à un banc de sable où ils étaient assommés, non sans que les oiseaux voletant, ailes pendantes entre les pieds des chevaux, provoquent sauts de mouton, cabrages et ruades des montures. 


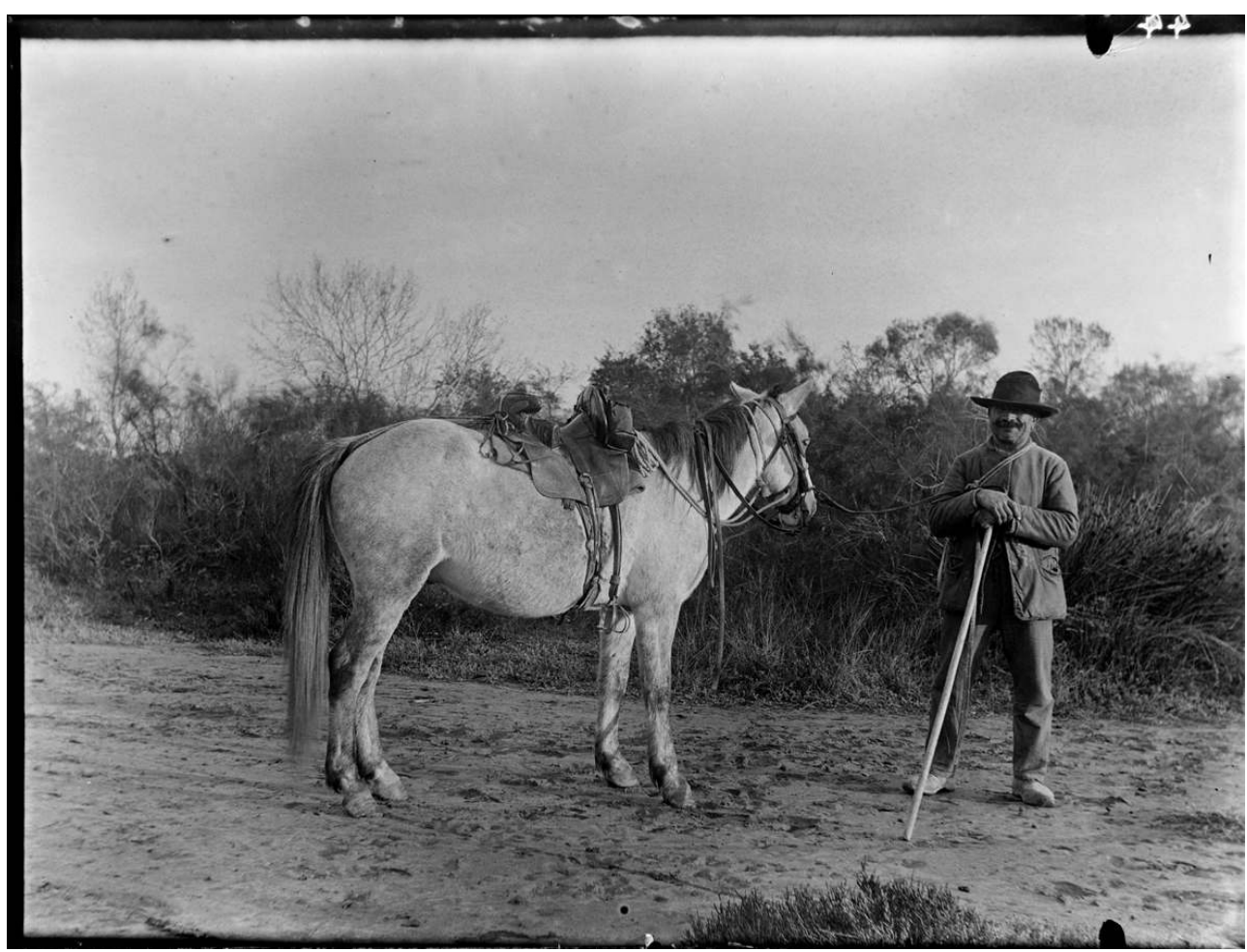

Gardian surveillant son troupeau « à bâton planté » vers 1910.

Phot. Naudot, Carle, coll. Musée de la Camargue, PNR de Camargue. Num. David Huguenin (8301795). (c) Musée de la Camargue, PNR de Camargue.

10 Au printemps, une attention particulière doit être portée aux femelles gestantes car la mise bas pourrait se dérouler dans un endroit défavorable et le poulain ou le veau risqueraient l'enlisement ou la noyade. Dans ce cas, le petit doit être récupéré avant d'être reconduit à sa mère. Il faut également surveiller les jeunes mâles qui ont tendance à beaucoup bouger. De nos jours, un apport complémentaire d'alimentation est fourni à ceux qui ont été sélectionnés pour les courses. La période estivale est consacrée aux fêtes votives pour lesquelles des lots de taureaux sont engagés, ceux-ci devant être séparés du troupeau et conduits aux arènes. Cette activité exceptionnelle sous l'Ancien Régime et dans la première moitié du XIX ${ }^{e}$ siècle ne s'est développée qu'avec la création des lignes de chemin de fer permettant au public de se déplacer vers les lieux de fêtes puis s'est largement étendue grâce au transport en camion des animaux. L'automne est l'époque du sevrage et traditionnellement, celle du marquage des veaux, avant que cette activité ne devienne, au milieu du $\mathrm{xx}^{\mathrm{e}}$ siècle, l'occasion d'une fête privée au sein des élevages, mais également l'objet de démonstration proposée aux touristes durant la saison estivale. À l'âge d'un an, le poulain, qualifié de court, était muni d'une sorte de caveçon, le salabre dont la muserolle en bois était munie de cinq pointes de fer qui en piquant le ventre de la mère provoquait des ruades obligeant à la séparation. Les veaux étaient équipés d'un mourrau, pièce de bois en forme de demilune suspendue par deux ergots à la cloison nasale, qui les empêchait de téter mais pas de brouter et qui tombait tout seul avec le temps. L'évolution de la zootechnie a permis l'abandon de ces pratiques. Les crinières des juments étaient coupées pour en recueillir les crins servant à la fabrication des seden, cordage d'environ $2 \mathrm{~m}$ de long, utilisé comme licol, longe ou lasso. L'hiver était la saison du débourrage des poulains: le 
gardian devait savoir débourrer et dresser les chevaux de manière à les rendre aptes à mener les troupeaux et à participer au travail de tri du bétail. Cette période de l'année était la plus pénible pour les troupeaux et les gardians. Les chevaux s'adaptent aisément à la froidure en revêtant une épaisse toison d'hiver, tournant le dos à la tourmente et cheminant lentement la tête basse. Un abri largement ouvert, la jasso leur était souvent destiné afin qu'ils puissent se soustraire quelque peu aux piqûres d'insectes en été et s'abriter du mistral en hiver. Ils passent la nuit serrés les uns contre les autres, couchant sur le sol boueux ou gelé. Les hivers plus rigoureux étaient surtout difficiles pour les juments gravides et provoquaient de fréquents avortements, ainsi que des problèmes de croissance pour les poulains. Par contre si le temps est trop mauvais, la manade de biòu se serre en se tournant du côté du vent et de la pluie : «vira la bano au giscle» et il peut advenir qu'en cas de grande froidure, les bêtes s'accroupissent et restent couchées tant que dure la tempête ; elles pourraient se laisser mourir d'inanition ou de froid si le gardian ne venait pas les remuer et les conduire dans un endroit plus abrité. Si en cas de gel, les chevaux sont capables de briser avec les pieds ou de ronger la glace pour boire, le gardian doit casser la glace recouvrant les points d'abreuvage et éventuellement deux fois par jour, pour que les bovins puissent s'y désaltérer.

11 La pratique du métier se faisait par transmission familiale, les fils des gardians rejoignaient leurs pères puis cette profession a attiré des jeunes gens passionnés parmi lesquels s'opérait une sélection drastique pour répondre aux rares offres d'embauche. L'apprenti gardian est désigné par le terme de gardianoun. Avec la conscription et le service militaire, les jeunes gardians furent généralement intégrés dans les compagnies de dragons puis suivant leur morphologie, dans des troupes de hussards ou de cuirassiers où ils trouvèrent l'occasion d'améliorer leurs connaissances équestres et leur pratique de l'équitation.

\section{Troupeaux de bovins}


Figure 5

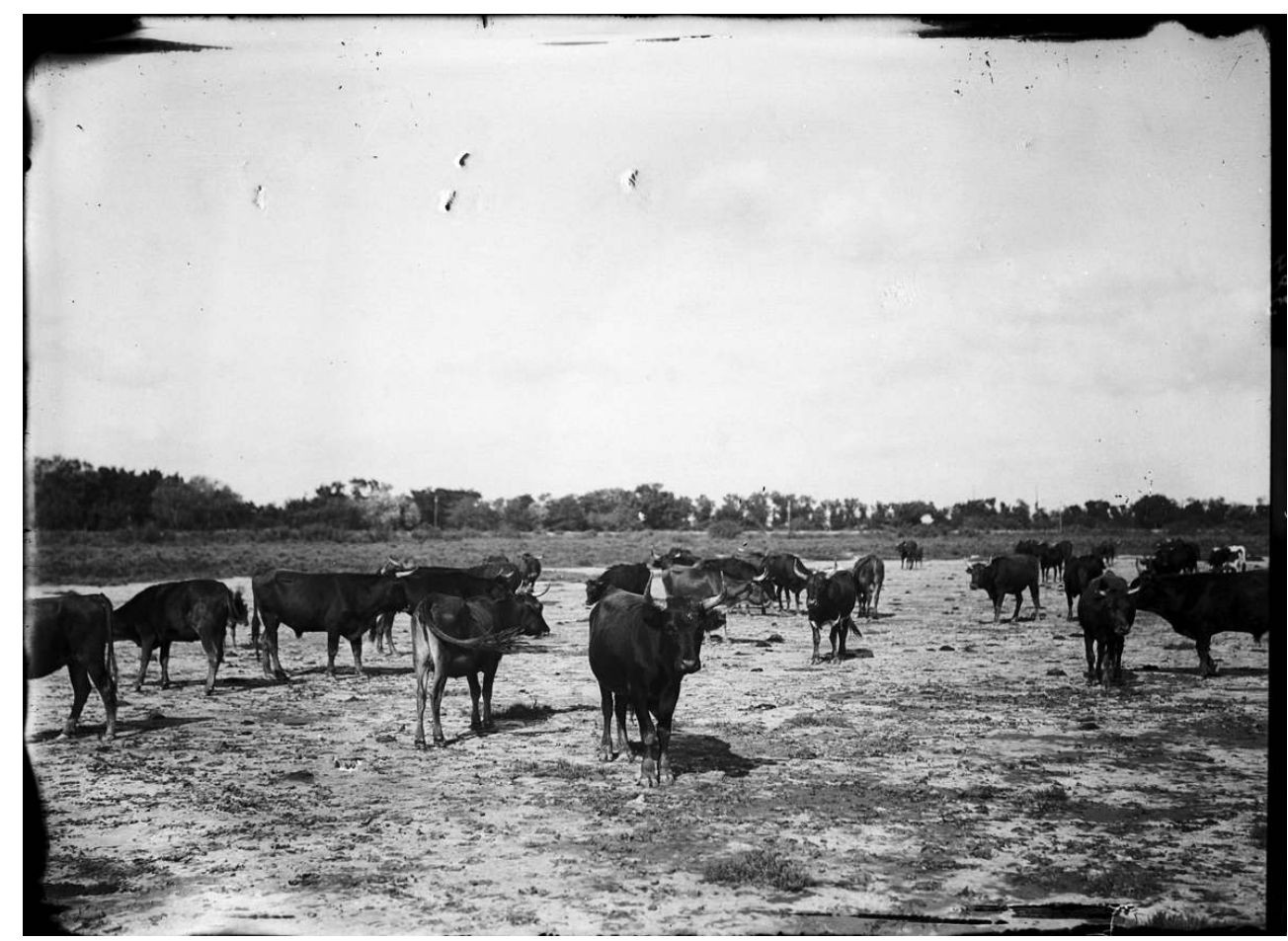

Manade de taureaux camargue avant 1948.

Phot. Naudot, Carle, coll. Musée de la Camargue, PNR de Camargue. Num. David Huguenin (8301616) (c) Musée de la Camargue, PNR de Camargue.

12 Le taureau de Camargue, la raço di biòu est imposant mais relativement léger. En moyenne les mâles mesurent $1,40 \mathrm{~m}$ pour un poids de $400 \mathrm{~kg}$ et sont plus grands et plus forts que les femelles qui toisent $1,20 \mathrm{~m}$ pour $250 \mathrm{~kg}$ (fig. 5). Leur caractéristique la plus remarquable réside dans les cornes, qui se dirigent verticalement vers le ciel en forme de lyre. Au milieu du Xvir ${ }^{e}$ siècle, le géographe Louis Coulon signale l'importance de l'élevage des taureaux et des chevaux dans l'île de Camargue et remarque que les taureaux y ont une fierté particulière ${ }^{5}$. L'agressivité naturelle du jeune taureau est matée par une action appelée « coup de fer » qui consiste à stopper la charge par un coup de fèrri ou ficheiroun : petit trident à pointes courtes, asséné sur le mufle (fig. 6). Ce geste particulièrement dangereux pour l'homme, surtout quand il est pratiqué à pied, fait l'objet de démonstrations lors de manifestations taurines et constituait le principal rite de passage au statut de gardian pour le gardianoun. 


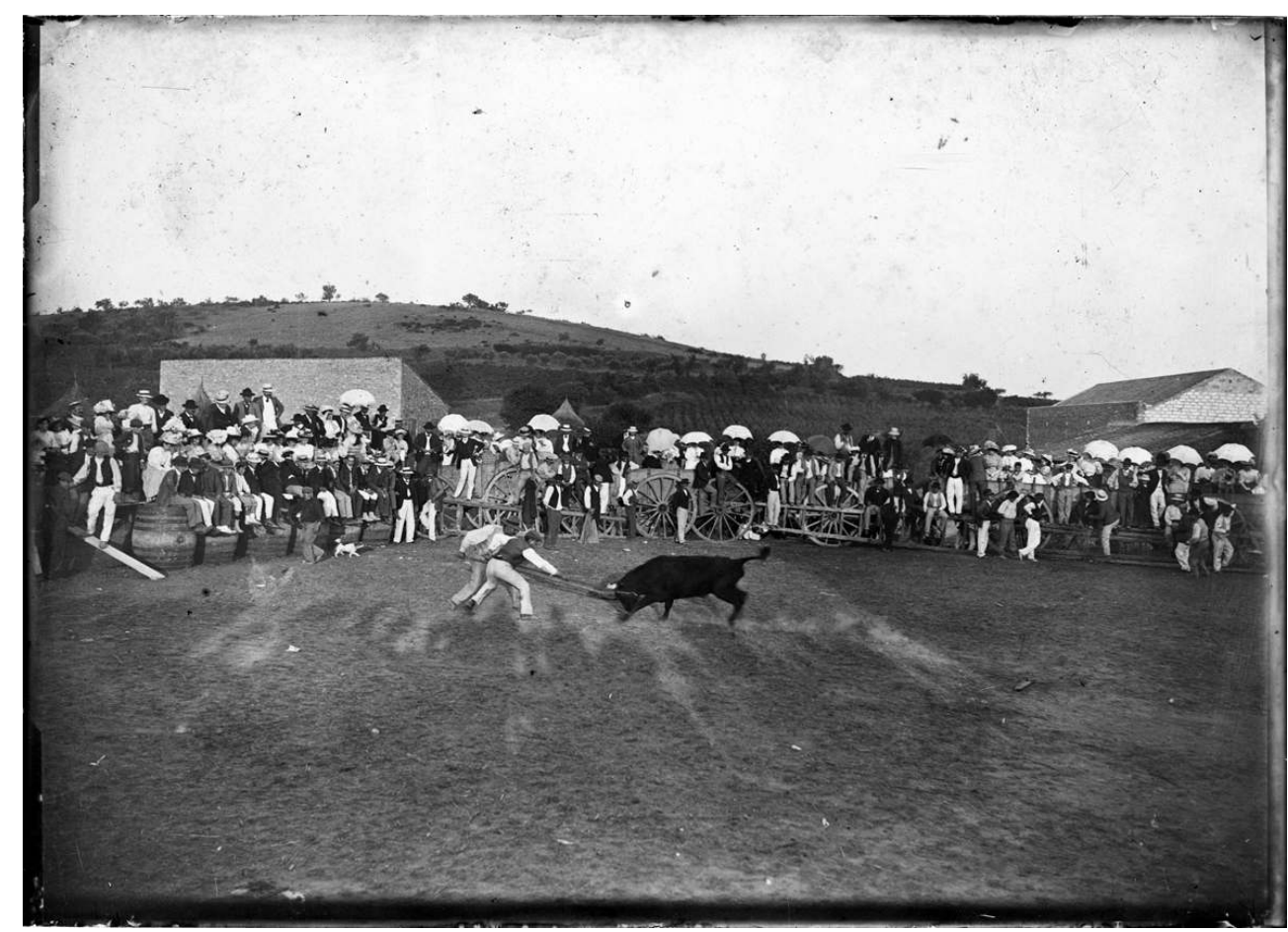

Attente au fer précédant la charge du taureau avant 1937.

Phot. Bouzanquet, Gaston, coll. Musée de la Camargue, PNR de Camargue. Num. David Huguenin (2010.01. 96). ( ) Musée de la Camargue, PNR de Camargue.

Bien qu'essentiellement élevés pour la viande, les bœufs fournissaient jusqu'au milieu $\mathrm{du} \mathrm{xv}^{\mathrm{e}}$ siècle, l'unique moyen de traction de l'araire utilisé sur les riches terres à blé arlésiennes, exploitées par assolement biennal et faisant l'objet de 5 ou 6 passages et même couramment de 8 labours profonds qui avaient cependant pour effet de remonter les couches inférieures salées et dépourvues d'humus en enfouissant la bonne terre superficielle formée par les limons du Rhône. Ils furent progressivement remplacés par les chevaux et surtout les mulets, entre 1780 et 1830. L'apprentissage de la traction des charrues est décrit par le baron de La Tour d'Aigues, en 1787 : les jougs de dressage étaient prévus pour associer trois animaux, d'un côté, un vieux bœuf docile et de l'autre, un autre bœuf expérimenté, le nouveau placé contre le timon était ainsi contraint de suivre le mouvement ${ }^{6}$.

En 1869, pour répondre à la demande des spectacles de tauromachie et présageant certainement l'évolution de la course camarguaise, des éleveurs ont introduisirent des taureaux de race espagnole et des croisements furent réalisés mais une préservation volontaire de modèles correspondant au standard actuel de la race camargue permit la sauvegarde du cheptel autochtone. La garde de ces nouveaux troupeaux s'effectuait cependant de la même manière que celle de la race locale par des gardians montant des chevaux camargue, purs ou croisés avec les étalons du dépôt d'Arles.

\section{Du travail collectif aux jeux taurins}

Si le gardian restait seul la plupart du temps pour la garde du troupeau, une partie du travail nécessitait d'avoir recours à un groupe de personnes, voire de cavaliers, plus ou 
moins important. Lors de la création de la confrérie professionnelle des gardians d'Arles, le 2 janvier 1512 et suivant l'esprit du temps, les principes énoncés étaient essentiellement à caractère religieux; cependant la fête annuelle s'articulait autour d'un repas collectif qui favorisait une rencontre entre collègues et au cours duquel ceux-ci devaient organiser leur programme annuel. Ainsi au XVII ${ }^{e}$ siècle, la confrérie tirait un revenu de la participation des gardians aux ferrades. Dès les premières années de la création de cette association professionnelle et de secours mutuel, quelques membres de la noblesse arlésienne en firent partie, montrant l'intérêt qu'ils portaient aux ressources de l'élevage.

Placée sous le patronage de saint Georges, la confrérie vient de fêter ses 500 ans d'existence. Très attachée au principe fondamental de secours mutuel entre ses membres, inhérent à ce type d'association, la confrérie respecte les statuts fondateurs par la présence des confrères lors des enterrements, la célébration annuelle d'une messe et une procession. Cette pérégrination urbaine devait se dérouler initialement à pied, par esprit d'humilité, mais dès le XviI ${ }^{e}$ siècle, alors que la possession d'un cheval n'est plus un marqueur social de l'aristocratie, les gardians défilent à cheval, sous la conduite d'un capitaine, affirmant ainsi la particularité de leur métier et associant leurs chevaux de travail à ce rite collectif de protection. À partir de cette époque, après le banquet des confrères, l'après-midi était consacrée à des courses de chevaux, dotées de prix, brides ou éperons offerts par la confrérie ; les gardians mesuraient également leur adresse à la course à la bague ou aux aiguillettes, jeu d'entraînement militaire où le cavalier doit viser un anneau suspendu à une potence et l'emporter sur la pointe d'une lance, qui remplaça pour la noblesse, les joutes interdites après la mort accidentelle du roi Henri II en 1559. Au XIX $x^{e}$ siècle, pour mettre en valeur les produits de l'élevage, des courses de chevaux étaient régulièrement organisées lors des fêtes locales.

Le déplacement des troupeaux de pâturage d'hiver en pâturage d'été et vice-versa exigeait l'encadrement de quelques cavaliers qui pouvaient être le pelot et le gardianoun ou quelques gardians du voisinage. Une sélection de bœufs, encadrée par des cavaliers, était périodiquement conduite vers les abattoirs. Au milieu du XIX ${ }^{\mathrm{e}}$ siècle, le manadier Yonnet menait régulièrement des troupeaux à Toulon, traversant Marseille de nuit. Ces parcours impliquaient parfois la traversée à la nage de cours d'eau, plus ou moins importants : dans le cas des Yonnet pour aller à Toulon, ils n'hésitaient pas à franchir le Grand Rhône près de son embouchure ${ }^{7}$. Cette pratique impressionnante, la gasado, francisée en "gaze» (fig. 7), donne lieu de nos jours à des manifestations spectaculaires appréciées du public. 


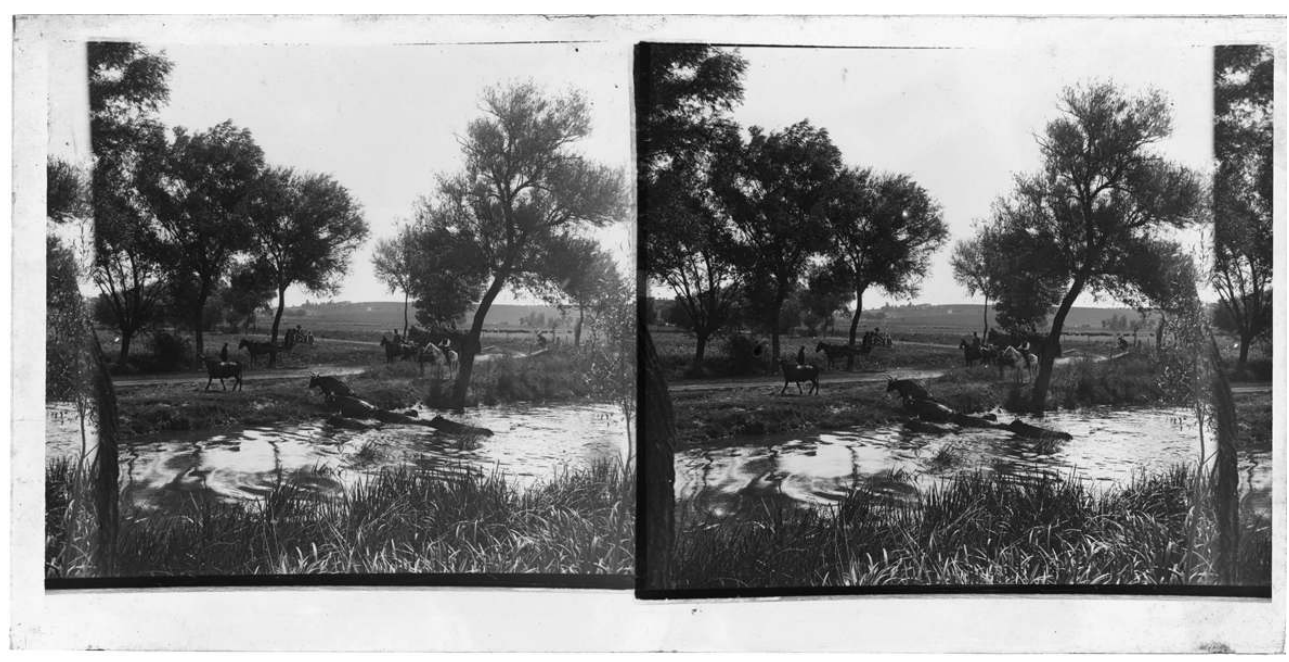

Gasado, franchissement d'un cours d'eau entre 1910 et 1925.

Phot. Bouzanquet, Gaston, coll. Musée de la Camargue, PNR de Camargue. Num. David Huguenin (2010.01. 37). @ Musée de la Camargue, PNR de Camargue.

Des spectacles mettant en valeur la combativité des taureaux de Camargue étaient coutumièrement organisés lors des grands événements marquant la vie de la cité arlésienne. La plus ancienne mention connue remonte à 1402 , lors de la visite du comte de Provence Louis II, où un taureau fut opposé au lion que la ville entretenait par tradition, ce félin étant l'emblème de la cité. Plus généralement, c'étaient des hommes qui s'opposaient aux taureaux lors de visites princières ou pour marquer des grands événements nationaux comme pour le couronnement de Louis XVI en 1775. Limitées à la ville d'Arles et à quelques villages des Alpilles, les courses de taureaux semblent avoir été beaucoup plus répandues dans les villages de Petite Camargue où elles ont firent l'objet de nombreuses interdictions préfectorales jusqu'à la naissance du roi de Rome (1811), occasion pour laquelle Napoléon leva les interdictions sur les réjouissances populaires interdites après la Révolution. Certaines bêtes achetées par un village ou une confrérie pour leur banquet faisaient l'objet d'un jeu particulier, le taureau à la bourgine : l'animal attaché par une longue corde enroulée autour de ses cornes était incité à la course par les plus téméraires. La finalité de cette pratique aurait été d'améliorer la qualité gustative de la viande car il s'agissait souvent de bêtes âgées, réformées par les laboureurs. Maintenue pour le jour de la fête de saint Bonnet (15 janvier), dans le seul village d'Eyragues (Bouches-du-Rhône), cette tradition qui a évolué et ne présente qu'un taureau aguerri à cette pratique, sous contrôle vétérinaire, et dont c'est la seule activité dans l'année, fait indûment l'objet d'attaques de la part des mouvements anti-corrida.

19 Un arrêt du conseil de la Ville d'Arles de 1715 interdit sous peine d'amende à toute personne de faire échapper les bœufs et les vaches conduits à la boucherie. De ces actes répréhensibles provient certainement la pratique de l'abrivado, conduite de taureaux encadrés par des cavaliers (fig. 8). Se déroulant généralement avant la course camarguaise qui a lieu dans des arènes, ce sont les six taureaux qui affronteront les hommes et qui sont placés sous la conduite d'un simbèu. Les cavaliers se disposent de manière à former un triangle enfermant les bœufs que des spectateurs s'évertuent à faire s'échapper en s'interposant entre les chevaux. 
Figure 8

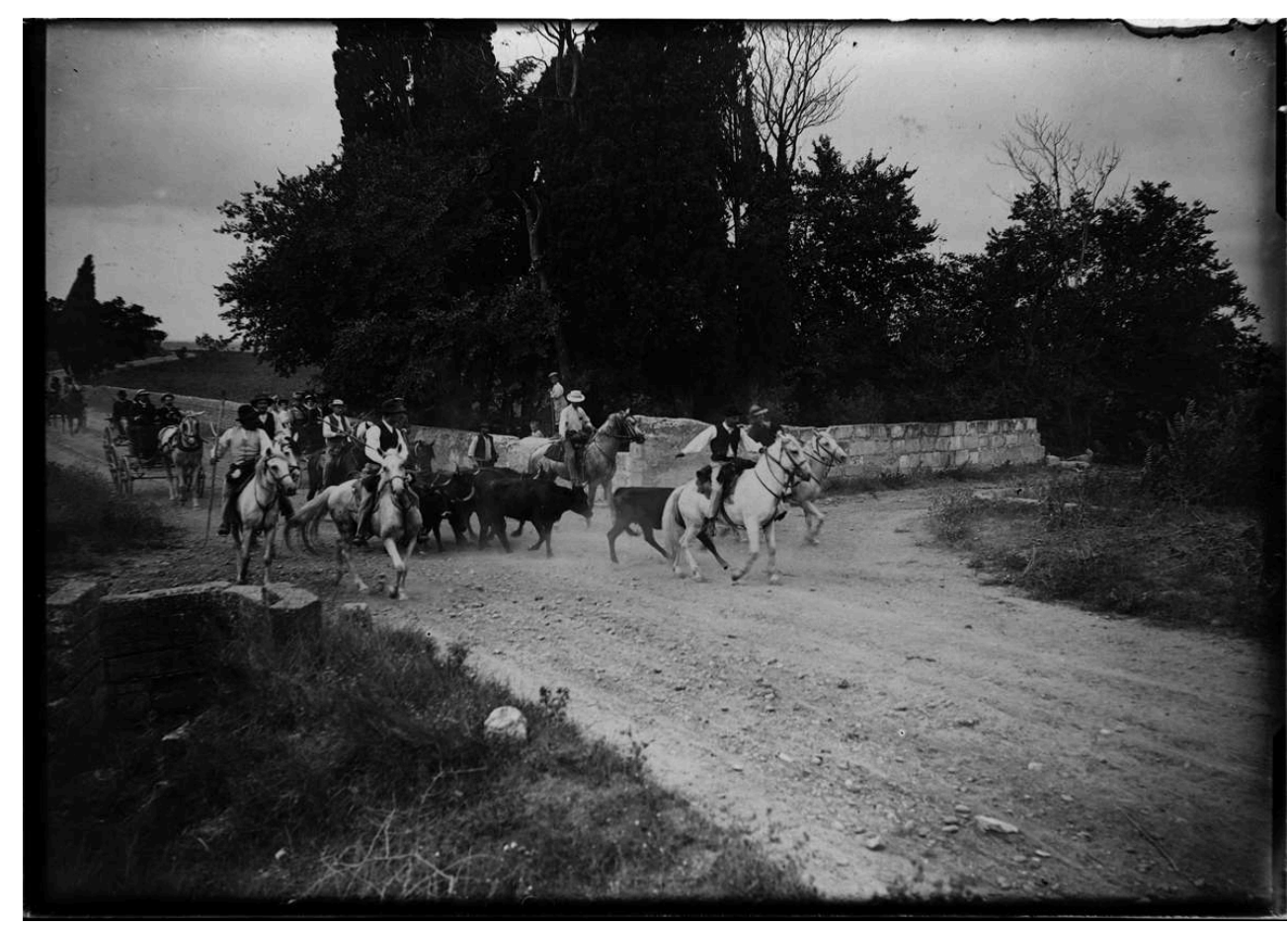

Abrivado ou conduite d'un troupeau entre 1910 et 1925.

Phot. Bouzanquet, Gaston, coll. Musée de la Camargue, PNR de Camargue. Num. David Huguenin (2010.01. 113). (C) Musée de la Camargue, PNR de Camargue.

Le marquage des bovins est l'objet d'une pratique particulière, la ferrado, ferrade revêtant une caractéristique sociale spécifique : les récits de voyageurs la signalent comme une curiosité et Louis Coulon, en 1643 précise que : « La noblesse a coustume de les combattre (les bœufs) à certains jours avec une grande solennité qu'ils nomment la ferrade $»^{8}$. Les propriétaires y conviaient effectivement de nombreux gentilshommes, certains venant cependant de leur propre initiative, mais également des gardians, des bouviers et des ouvriers qui arrivaient en nombre pour prêter main forte. On peut penser que les jeunes nobles y trouvaient l'occasion d'exercices équestres bénéfiques tant pour le cavalier que pour le cheval et qu'elle constituait un substitut aux chasses à courre peu pratiquées dans une région où le gros gibier était rare, tandis que les ouvriers agricoles faisaient montre de leur courage et de leur maitrise de l'animal (fig. 9). Le déroulement d'une ferrade au XvII ${ }^{\mathrm{e}}$ siècle est décrit par Pierre Quiqueran de Beaujeu qui précise qu'alors, l'événement ne se déroulait que tous les deux ou trois ans pour chaque troupeau et concernait ainsi des bovillons de différents âges : anouble, doublen et tercen, suivant qu'ils aient un, deux ou trois ans ${ }^{9}$. 


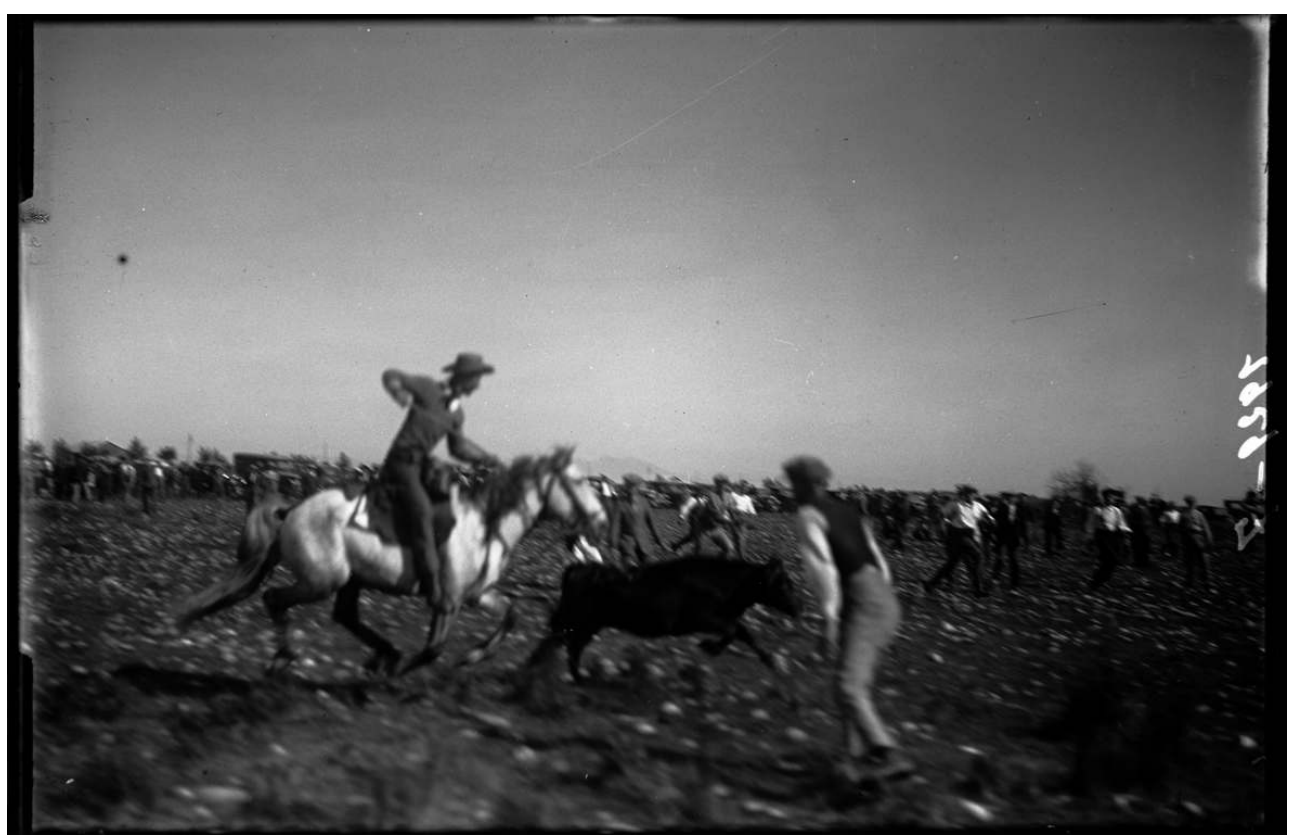

Ferrado ou marquage au fer chaud en 1929.

Phot. Naudot, Carle, coll. Musée de la Camargue, PNR de Camargue. Num. David Huguenin (83011201). (C) Musée de la Camargue, PNR de Camargue.

21 La manade était rassemblée à l'extrémité d'une grande prairie, bien plate, sèche, sans pierres ni ronces. Un enclos ouvert provisoire était aménagé à l'autre extrémité et un feu où les fers aux chiffres et marques des maîtres étaient mis à rougir, était entretenu à l'autre extrémité. Les gardians séparaient les bêtes sélectionnées et les poursuivaient individuellement jusqu'au groupe de piétons stationnant près du feu, sur un parcours suffisamment long pour «éviter que le gros du troupeau soit spectateur du mauvais traitement qu'on fait à leurs frères, sinon saisis d'effroi, ils s'enfuiraient » mais surtout pour fatiguer l'animal par la course-poursuite. Près du feu, un coup de trident asséné perpendiculairement, à la hauteur de la naissance de la queue permet de renverser le veau. Ce geste nécessitant les deux mains, le cheval doit continuer sa course sur sa ligne de lancée, la bride relâchée puis s'arrêter net une fois l'action effectuée. Les plus intrépides parmi les piétons s'emparaient alors promptement de la corne gauche et de la patte antérieure droite et en poussant d'un coup d'épaule, l'animal était jeté au sol « d'une si rude secousse que la terre résonne sous le choc». De nos jours, les gardians poursuivant un veau mettent un point d'honneur à réaliser entièrement l'opération, descendant de cheval pour attraper l'animal renversé. Le veau était alors marqué au fer et à moins d'avoir été sélectionné comme reproducteur, il était bistourné, c'est-à-dire castré par torsion des canaux séminaux. L'animal subit encore une découpe du cuir des oreilles, l'escoussuro, marque distinctive particulière à chaque manade.

L'agressivité de l'animal libéré était déjà une première occasion d'amusement pour les participants mais après l'inévitable festin qu'offrait le propriétaire, les gardians allaient chercher le plus farouche taureau afin de le mener vers les piétons pour les divertir, la bête renversant et jetant à terre tous ceux qui venaient à sa rencontre. Les amateurs recherchaient ce genre de sensation forte et tiraient une grande fierté d'avoir été bousculés voire renversés. De ce comportement agressif du taureau découlent les principes de la course camarguaise, pratiquée d'abord dans les cours des mas, dans des 
arènes improvisées par des charrettes disposées en cercle, mais surtout lors des fêtes votives, puis organisées par les clubs taurins. La course camarguaise fait aujourd'hui, l'objet de compétitions sportives, et dans un triangle constitué par Avignon au nord, Marseille à l'est et Montpellier à l'ouest, chaque village dispose d'arènes où se déroulent les spectacles taurins.

\section{Troupeaux de chevaux}

Le cheval camargue est un animal robuste, toisant entre $1,35 \mathrm{~m}$ et $1,48 \mathrm{~m}$ et pesant entre 300 et $400 \mathrm{~kg}$ à l'âge adulte, suivant le standard contemporain. Bien qu'actuellement, ils soient caractérisés par leur robe «blanche », les chevaux camargue jusqu'au $\mathrm{xx}^{\mathrm{e}}$ siècle, pouvaient avoir des robes bai, alezan ou noire; la sélection de spécimens à robe grise est attribuée au fait que ces animaux sont moins assaillis par les différents insectes piqueurs pullulant dans les marécages ${ }^{10}$. Une volonté d'uniformisation des groupes de cavaliers et une opposition à la robe noire des taureaux ont cependant pu déterminer cette sélection dans un but esthétique. Rustique, maniable et endurant, le cheval de Camargue a un excellent sens de l'orientation et une bonne connaissance de la nature des sols; le gardian s'en remet facilement à ses réactions instinctives pour apprécier les dangers et les éviter. Se déplaçant avec aisance sur tous types de terrains, en particulier dans les marais et les fonds vaseux, il a un pas très rapide, proche de l'amble, travaille au tri du bétail, le plus souvent au trot et passe instantanément au galop par des démarrages rapides et peut facilement volter à toutes les allures. Pour démontrer les qualités d'endurance du camargue, le marquis de Baroncelli réalisa un raid en mars 1905, partant à deux cavaliers montés sur des chevaux l'un de 14 ans et l'autre de 8-9 ans. Ils relièrent Lyon, par la rive gauche, en 4 jours et demi et après une journée et demie de repos, ils redescendirent par la rive droite en autant de temps, soit $630 \mathrm{~km}$ au pas, à $7 \mathrm{~km} / \mathrm{h}$, avec comme plus longue étape Montélimar-Saint-Vallier, soit $82 \mathrm{~km}$ et 11 heures en selle. Les chevaux qui étaient tout juste sortis des pâtures d'hiver, rentrèrent en parfait état. Le cheval camargue n'hésite pas à nager dans les roubines en eau ou même à traverser les bras du Rhône : plusieurs récits de sauvetages relatent le courage de gardians et de leurs chevaux, qui, au cœur des tempêtes, effectuaient des navettes pour évacuer les naufragés de bateaux échoués sur le littoral. Malgré le dressage, le cheval camargue garde le caractère sauvage que lui confère sa jeunesse en liberté, ce qui lui valut au XVII siècle, la dénomination de « rosse » dans son acceptation ancienne de cheval farouche, avant que ce terme désigne un mauvais cheval, rétif ou malformé.

Qu'au XvI ${ }^{\mathrm{e}}$ siècle, le gardian spécialisé dans la garde des troupeaux d'équidés ait été plus particulièrement désigné par le terme d'egatié ou par l'expression équivalente de gardian d'ego, soit de gardian de juments, peut s'expliquer par le fait que le prélèvement des jeunes mâles pour leur utilisation était systématique. Seul l'étalon, le grignoun ou garagnoun, un sujet de qualité mais difficile à utiliser et toujours laissé sauvage, restait avec la harde de femelles, ce qui avait pour effet de le rendre particulièrement agressif envers d'autres mâles même castrés (fig. 10). La monte se pratiquait en liberté et aucun soin particulier n'était donné aux juments pleines. Une surveillance des mises bas était cependant nécessaire, en particulier pour les juments primipares, souvent jeunes et inexpérimentées. Après la période des naissances, les juments étaient soumises à une tonte de la crinière dont les crins servaient à la confection des seden, activité artisanale 
que les gardians pratiquaient en hiver, conjointement à la fabrication de cloches et de mourrau. Si les juments âgées avaient fini par s'habituer, les plus jeunes se montraient particulièrement rétives à cette opération.

Figure 10

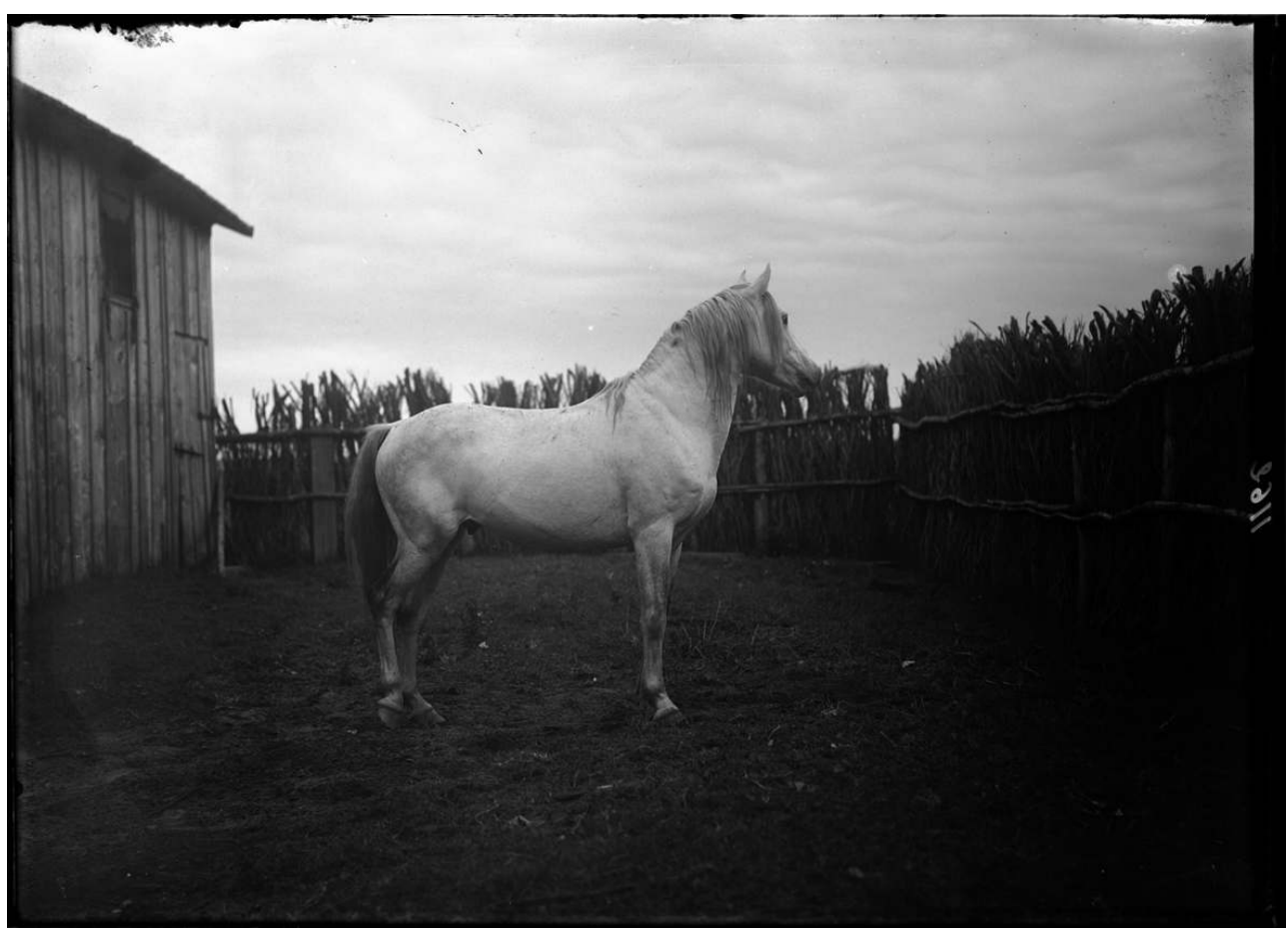

Étalon camargue.

Phot. Naudot, Carle, coll. Musée de la Camargue, PNR de Camargue. Num. David Huguenin (8301710). (c) Musée de la Camargue, PNR de Camargue.

Les chevaux camargue ont principalement été utilisés comme chevaux de selle, pour le travail des gardians mais également pour tout type de cavaliers. Au XII siècle, la cité d'Arles fournit 300 chevaux au comte Raimond Bérenger, dont une partie au moins devait être issue de l'élevage local. Si la morphologie des chevaux de Camargue du bas Moyen-Âge était proche de celle que l'on peut entrevoir au début de l'époque moderne, ils pouvaient constituer des montures tout à fait acceptables pour des chevaliers, au moins jusqu'à l'apparition des armures de plates. Dès 1529, la communauté d'Arles institua une course annuelle pour distinguer les bons produits et les meilleurs élevages. Pierre Quiqueran de Beaujeu reconnaît que la morphologie du camargue, considéré de taille moyenne, ne correspond plus aux critères physiques recherchés au milieu du xvie siècle, surtout à cause de son "manque de prestance ", il n'en vante pas moins les qualités de la race: légèreté, vigueur, fougue, rapidité, maniabilité, résistance et rusticité $^{11}$. Des chevaux camargue ont été achetés pour servir des montures à des cavaliers des troupes royales durant les guerres de Religion. L'introduction de chevaux améliorateurs, normands et limousins, sous le règne de Louis XIII, se solda par un dépérissement de ces reproducteurs inadaptés aux rigueurs du climat local. Sous le règne de Louis XIV, certains meneurs de bandes de camisards, étant originaires de Petite Camargue, assurèrent la remonte de leurs troupes avec des chevaux de Camargue. Arles fut doté d'un dépôt d'étalons des haras de Provence en 1729 mais quand en 1796, le $22^{\text {e }}$ régiment de chasseurs engagé dans les guerres d'Italie acheta des chevaux camargue, 
ce fut pour les utiliser comme chevaux de bât car ils ne convenaient plus aux cavaliers d'alors. Au cours de la première moitié du XIX ${ }^{\mathrm{e}}$ siècle, cependant les haras poursuivirent leurs efforts pour augmenter de la taille des chevaux camargue auxquels ne manquaient que quelques centimètres pour faire l'affaire des hussards et des chasseurs.

À l'âge de 2 ans, le poulain, qualifié de doublen, était marqué au fer de la marque de l'éleveur et au cours de sa troisième année, devenus ternen, ceux qui n'étaient pas sélectionnés pour la reproduction étaient castrés. Ces opérations se déroulaient dans le cadre privé de la cour des mas ; contrairement au bistournage des taureaux, l'opération s'effectuait dans l'intimité. La manade était enfermée dans un enclos puis les jeunes chevaux étaient attrapés au lasso, couchés et immobilisés par une demi-douzaine d'hommes (fig. 11). Avant que la castration ne soit réalisée par un vétérinaire, c'était un gardian expérimenté qui était sollicité. Aux XVIIIe et XIXe siècles, des professionnels piémontais ou béarnais pouvaient être spécialement engagés pour cette intervention. L'opération était effectuée avec des pinces ou plus généralement à l'aide de casseaux, baguettes de bois placées de manière à comprimer les cordons séminaux.

Figure 11

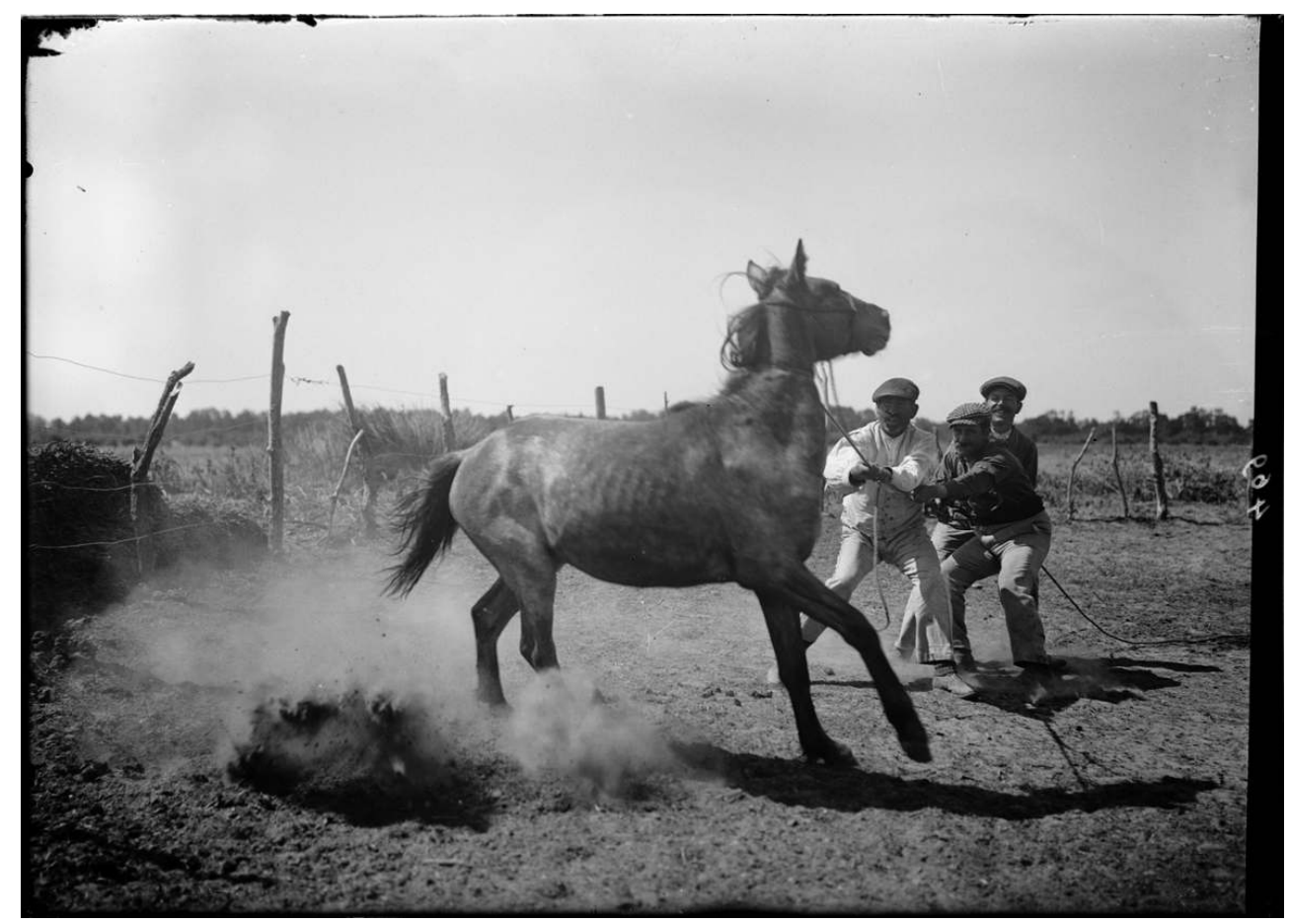

Capture au nœud coulant vers 1920.

Phot. Naudot, Carle, coll. Musée de la Camargue, PNR de Camargue. Num. David Huguenin (8301691). (c) Musée de la Camargue, PNR de Camargue.

27 Laissé en liberté jusqu'à l'âge de 4 ans, le jeune cheval était, après une approche prudente, attrapé individuellement grâce à une corde passant dans un nœud coulant et placée de manière à former une muserolle autour du chanfrein, ce qui permettait de limiter les réactions énervées de l'animal qui allait rejoindre le groupe des chevaux dressés, la principale défense du camargue étant les sauts de mouton. Le débourrage se faisait en hiver, les auteurs n'étant pas d'accord sur la méthode employée, qualifiée par certains de brutale; par d'autres de respectueuse de la nature du poulain ${ }^{12}$. Le jeune cheval était d'abord initié, non sans réticences, aux saveurs de l'avoine, ce qui facilitait 
par la suite le contact lorsque le gardian allait le chercher au pré car même dressé, le cheval ne se laissait pas attraper autrement. Le dressage se poursuivait par une accoutumance au harnachement, bride et caveçon et au port de la selle puis par un travail à la longe préparant à la monte par un cavalier. Il était d'abord conduit à pied puis par un homme monté sur un cheval expérimenté : le jeune cheval était alors initié aux exercices de rênes aux trois allures (fig. 12). Ce processus de débourrage traditionnel est proche de celui décrit par Jordanus Rufus, maréchal de Frédéric II de Hohenstaufen au XIII ${ }^{e}$ siècle $^{13}$. Au XvII ${ }^{e}$ siècle, Pierre Quiqueran de Beaujeu signale que seuls quelques gentilshommes, experts en dressage, formaient de bons chevaux pour la selle ${ }^{14}$. Il était recommandé de ne desseller le cheval qu'une heure après la session de travail. La sangle en cuir des selles gardiannes est maintenue par un nœud et non par une boucle ; en règle générale, le cheval n'est jamais désanglé tant que sa journée n'est pas terminée, cette pratique étant censée conférer une attitude patiente aux montures. Les particularités du travail demandé au cheval dans le cadre des manades de Camargue ont conduit à la théorisation de la monte gardianne, qui a été reconnue par la Fédération Française d'équitation en 1995.

Figure 12

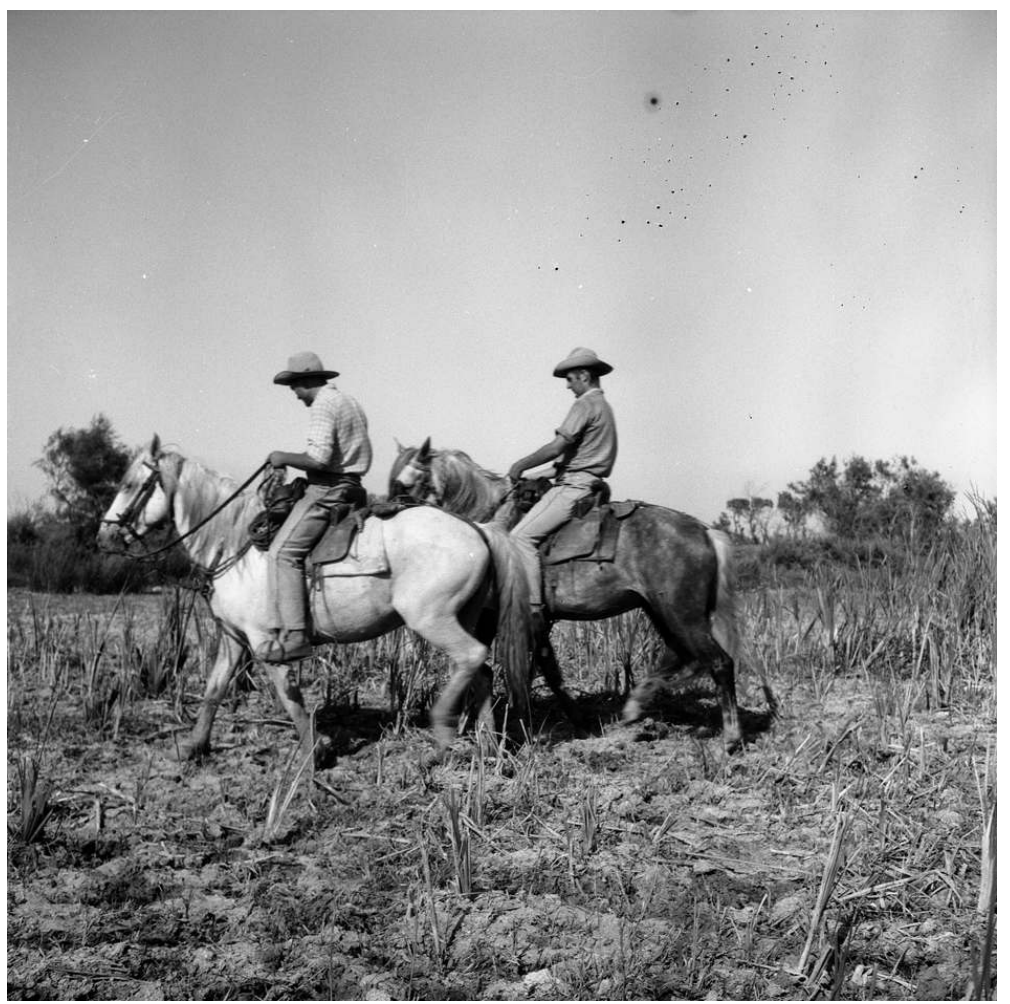

Phase de l'éducation par l'exemple en 1947.

Phot. Naudot, Carle, coll. Musée de la Camargue, PNR de Camargue. Num. David Huguenin (8301728.1). (c) Musée de la Camargue, PNR de Camargue.

Pour l'apprentissage du jeune cheval, douceur, patience et compréhension étaient particulièrement recommandées mais peu de chevaux avaient les qualités recherchées pour la garde des troupeaux : un bon cheval de gardian connaît son travail, sait se placer et rassemble, arambe, le troupeau à la manière d'un chien de berger ; un gardian sait le ménager pour lui permettre une longue carrière. Un apprentissage du travail, estimé à deux ou trois années après le débourrage, était nécessaire pour obtenir un 
cheval dit «à main finie » mais à condition que celui-ci bénéficie d'un sens particulier inné. Des lignées équines constituaient un trésor caché dans certaines manades, éventuellement soustraites aux contrôles de l'administration des Haras car ce « sens du taureau » dont faisaient preuve certains chevaux était mis sur le compte de l'hérédité, même si isolément tel individu pouvait se révéler soit médiocre soit exceptionnel. La mémoire collective conserve l'expression de chevaux « ratés ", peut-être mal débourrés et ombrageux mais probablement dépourvus des capacités requises pour le travail demandé. C'est au cours du débourrage que le jeune cheval était dénommé, suivant un qualificatif illustrant son caractère. Un bon cheval de gardian pouvait faire une carrière de 25 ans, avant d'être définitivement relâché dans la manade jusqu'au terme de sa vie.

Lié à la vie quotidienne du gardian, le cheval fut associé aux différentes pratiques religieuses intimes dès le $\mathrm{xIX}^{\mathrm{e}}$ siècle : il participait au cortège et à la haie d'honneur des mariages, conduisait le nouveau-né dans les bras de sa marraine pour les baptêmes et suivait le cortège funéraire de son propriétaire, la selle recouverte d'un crêpe noir. À leur mort, certains chevaux particulièrement méritants ont même été enterrés au cœur de la manade, disposés debout et équipés de tout leur harnachement. D’autres chevaux fatigués ou âgés servaient pour la formation des gardianoun, leur permettant de rejoindre le mas en fin de journée, mais généralement sans selle et au pas (fig. 13).

Figure 13

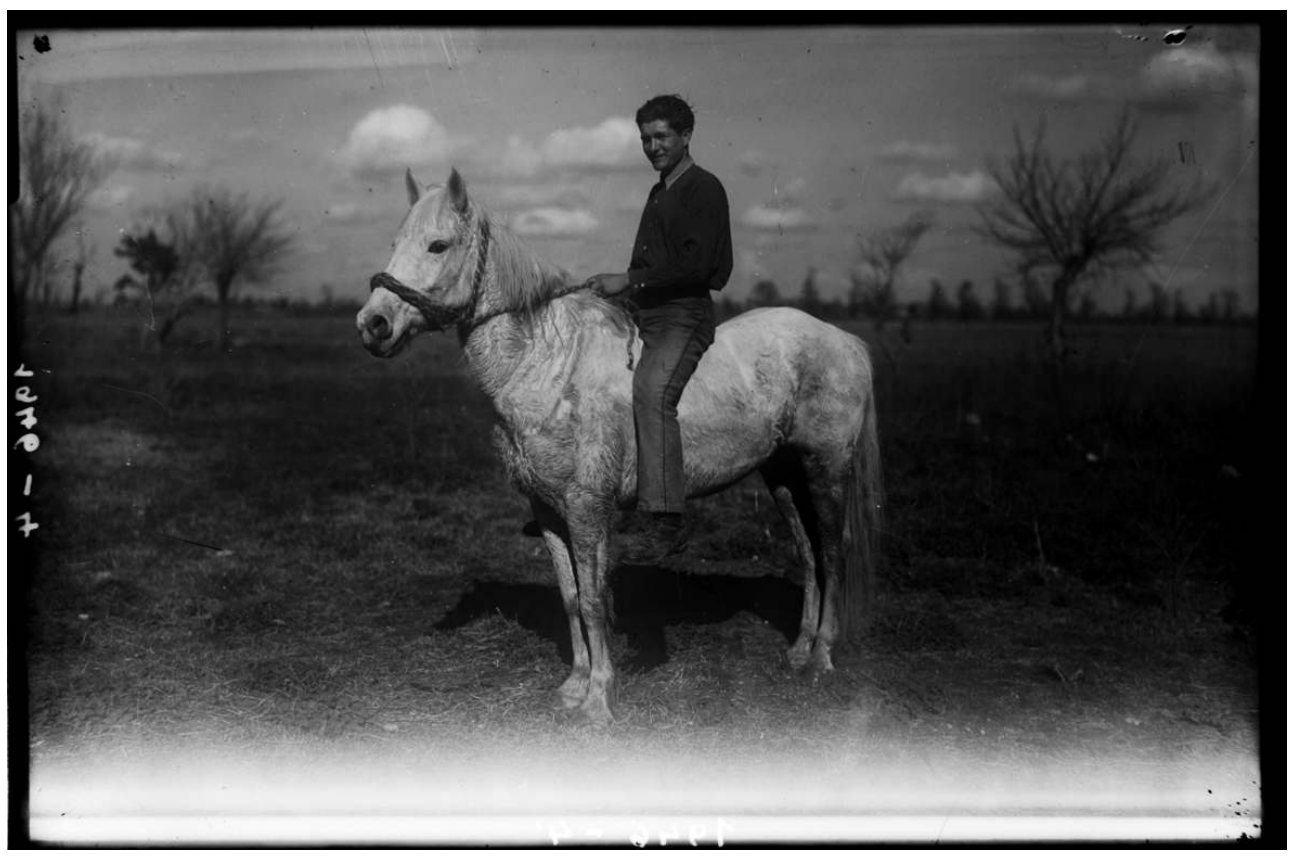

Hubert Yonnet, jeune gardian en 1946.

Phot. Naudot, Carle, coll. Musée de la Camargue, PNR de Camargue. Num. David Huguenin (8301769) (c) Musée de la Camargue, PNR de Camargue.

30 Grâce à une sélection, certains animaux étaient orientés vers l'attelage dont l'apprentissage était plus facile, le débutant étant associé à un cheval confirmé. Mais souvent, un même cheval, qualifié de voiturier, servait également pour la selle. Un bon cheval camargue sait mener son attelage et le sortir de situations délicates sans sollicitation de la part du conducteur. Dans les chemins détrempés avec de la boue jusqu'aux moyeux, le camargue arrive à se tirer d'affaire pourvu qu'on le laisse régler 
seul l'effort à fournir. Dès le $\mathrm{xvI}^{\mathrm{e}}$ siècle, des gardians sont désignés par le qualificatif de «carretier ». L'isolement des habitants de Camargue était interrompu par le marché du samedi matin à Arles, où les pelot se rendaient en charrette, souvent des jardinières, pour y traiter leurs affaires et éventuellement pour recruter des ouvriers agricoles (fig. 14). Attelés à la charrette comme à la charrue, les chevaux de Camargue trouvèrent ainsi un important débouché en particulier auprès de commerçants comme les poissonniers de Saint-Gilles (Gard), des agriculteurs et surtout des vignerons des petits villages du Languedoc oriental.

\section{Figure 14}

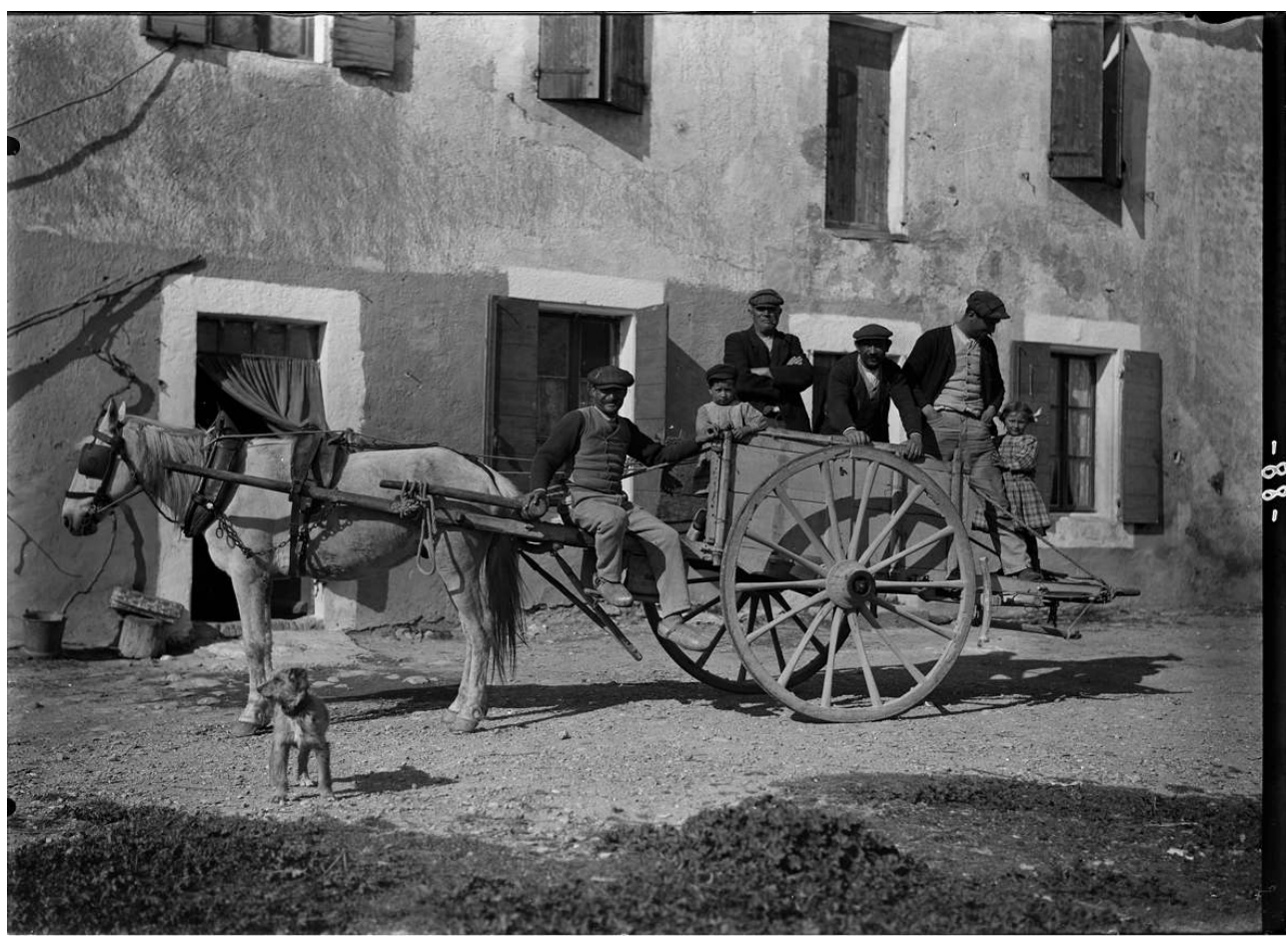

Cheval camargue attelé à une jardinière entre 1910 et1925.

Phot. Naudot, Carle, coll. Musée de la Camargue, PNR de Camargue. Num. David Huguenin (8301576) (c) Musée de la Camargue, PNR de Camargue.

31 Le territoire d'Arles produisant surtout du blé, les chevaux, exceptionnellement des mules mais plus spécifiquement les juments, furent utilisés pour le dépiquage des récoltes (fig. 15). Les gerbes étaient placées verticalement sur le pourtour d'une aire en terre battue, rarement pavée, le conducteur de roue menait douze bêtes attachées par paire, les liame dont la première et la dernière étaient équipées de colliers de cloches. Placé au centre, le conducteur, tenant les six longes, faisait tourner les chevaux, qui en piétinant les gerbes en séparaient les grains. Ce travail, qui l'on accomplit jusqu'à l'arrivée des batteuses à vapeur, à la fin du XIX siècle, était effectué au mois d'août, dans la chaleur et la poussière, du matin au soir et pendant plusieurs jours d'affilée, contraignant les chevaux à effectuer un parcours quotidien de 70 à $90 \mathrm{~km}$. Lantelme de Romieu au XVI $\mathrm{e}^{\mathrm{e}}$ siècle, signale des roues composées de 9 paires de juments et même des roues de cinquante à soixante animaux travaillant de manière autonome, sans conducteur, permettant une exécution rapide de la tâche ${ }^{15}$. 


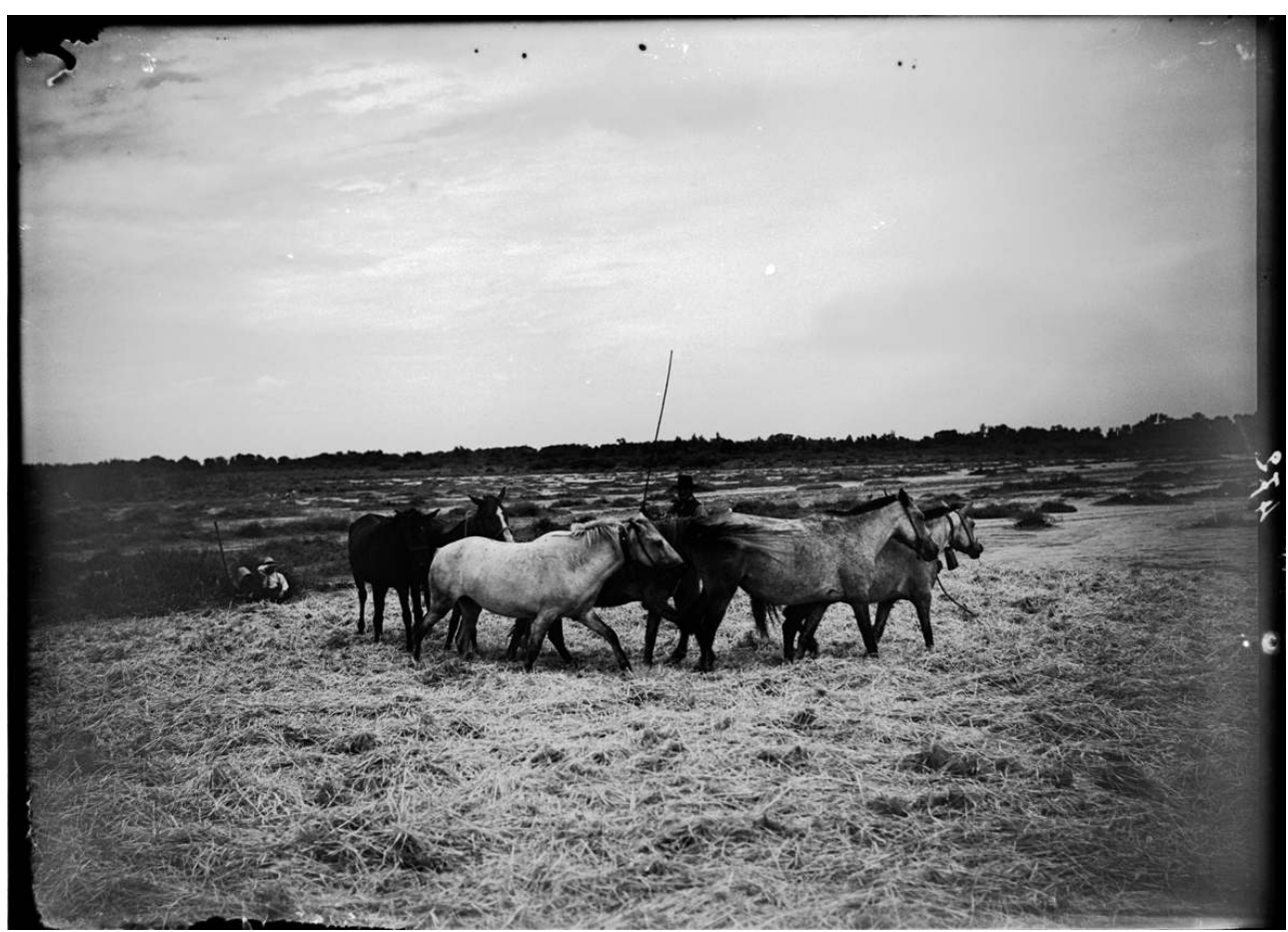

Roue de dépiquage vers 1910.

Phot. Naudot, Carle, coll. Musée de la Camargue, PNR de Camargue. Num. David Huguenin (8301821) (c) Musée de la Camargue, PNR de Camargue.

\section{Conclusion}

Issu des élevages aristocratiques et ecclésiastiques du bas Moyen-Âge et particulièrement adapté à son milieu naturel, le cheval de Camargue est essentiellement reconnu pour son aptitude à la garde des troupeaux de bovins et de chevaux laissés en semi-liberté au sein des grandes zones de pâturages marécageux du delta du Rhône. C'est le partenaire indispensable du gardian, pasteur cavalier de ce cheptel isolé au milieu de grands espaces sauvages. Documenté à partir du $\mathrm{XVI}^{\mathrm{e}}$ siècle, soit à la suite de transformations du mode de gestion territoriale de la Camargue, le métier de gardian n'a semble-t-il connu que peu d'évolution jusqu'au début du $\mathrm{xx}^{\mathrm{e}}$ siècle. Si la capture des poulains était plutôt rude, le mode de débourrage pratiqué était proche de celui décrit dès le XIII ${ }^{e}$ siècle préconisant patience et douceur pour un meilleur apprentissage. Une sélection drastique était opérée parmi les sujets car peu étaient réellement aptes au travail de conduite de troupeaux et du tri du bétail mais une fois les principes acquis, le cheval du gardian participait activement à la tâche, connaissait une longue carrière avant de jouir d'une retraite paisible ; il bénéficiait d'un statut à part au sein de la famille en étant associés aux cérémonies. Les sujets qui n'étaient pas sélectionnés trouvaient un débouché pour la selle ; en dépit de leur petite taille ils servirent dans des corps de cavalerie jusqu'au xviII ${ }^{e}$ siècle, et furent aussi employés pour l'attelage dans l'agriculture et le transport. 


\section{BIBLIOGRAPHIE}

AUBERT, François. La race chevaline Camargue. Nîmes : imprimerie Larguier, 1939.

BARONCELLI, Folco de. L'élevage en Camargue : le taureau (1931). Nîmes : L’Aucèu libre, 2007.

BARONCELLI, Folco de. Raconte camarguen - Récits camarguais (1935). Nîmes : L'Aucèu libre, 2003.

BERRIOT, Christian. Le cheval de Camargue. Lyon : imprimerie Bosc frères, 1969.

BERTRAND, Régis. «La fête provençale, du préfet Villeneuve à Mistral ». Dans BERTRAND, Régis et FOURNIER, Laurent-Sébastien (dir.). Les fêtes en Provence autrefois et aujourd'hui. Aix : Presses universitaires de Provence, 2014, p. 20.

BLONDEL, Jacques, BARRUOL, Guy et VIANET, Régis (dir.). Encyclopédie de la Camargue. Paris : éditions Buchet Chastel, 2013.

BRUGUIÈRE, Patrick. Une tradition régionale : les origines de la course libre 1800-1852. Nîmes : CIAM, 1996.

CHEVALIER, Annelyse. «Les gardians de Camargue ». Courrier du parc naturel régional de Camargue, $n^{\circ}$ 56, 2007.

COULON, Louis. L'Ulysse françois ou le voyage de France, de Flandre et de Savoye. Paris : chez Gervais Clousier, 1643.

DIGARD, Jean-Pierre. Une histoire du cheval, art, techniques, société. Arles : Actes Sud 2007.

Du LAC, Marc. Le cheval camargue. Arles : Actes Sud, 1999.

DURAND, Jacques. André Bouix, gardian de Camargue. Paris : Stock, 1980.

GADIOT, Gérard. En Camargue : taureaux, chevaux et gardians. Paris : Arthaud, 1977.

GALLON, Cécile. La confrérie Saint-Georges des gardiens d'Arles de 1634 à 1782. Nîmes : Sansouire, 2012.

LABORIEUX, Alain. Des siècles de bouvine, une approche historique de la tauromachie provençale et languedocienne. Lunel-Vieil : Espace Sud, 2002.

LANGLOIS, Bertrand. « Le gardian de Camargue entre mythes et réalités ; entretiens avec JeanClaude Gleize ». Ethnozootechnie, 2007 nº 80, p. 63.

MISTRAL, Frédéric. Lou tresor dóu Felibrige : dictionnaire provençal-français. 2 tomes. Aix-enProvence : Édisud, 1979.

NAUDOT, Carle. Camargue et gardians : ethnographie folklorique du pays d'Arles. Arles : Parc naturel régional de Camargue, 1989.

NAUDOT, Carle et BOUZANQUET, Gaston. Objectifs croisés sur la Camargue. Arles : Actes Sud/Parc naturel régional de Camargue, 2010.

QUIQUERAN de BEAUJEU, Pierre. Louée soit la Provence (1551). Adaptation par Véronique Autheman. Arles : Actes Sud, 1999.

RAYNAUD, Frédéric. « La confrérie des gardians d'Arles 1512-1519 ». Bulletin des Amis du Vieil Arles, 2014, n 159, p. 39-58.

ROSSIGNOL, Ferdinand. Les Protestants illustres : portraits-biographies. Paris : C. Meyrueis et Cie, 1863. 
ROUQUETTE, Jean-Maurice (dir.). Arles : histoire, territoires et cultures. Paris : Imprimerie nationale, 2008.

RUFUS, Jordanus. La science du cheval au Moyen-Âge, le traité d'hippiatrie de Jordanus Rufus. Éd. Brigitte Prévot. Paris : Klincksiek, 1991.

RUL, Élie. La Camargue Gardianne. D’Elly. Paris : éditions M. Delaveau, 1938.

SAUMADE, Frédéric. « Le marquis de Baroncelli et Buffalo Bill spectacles du sauvage et fêtes tauromachiques en Camargue et en Amérique du Nord ». Dans BERTRAND, Régis et FOURNIER, Laurent-Sébastien. Les fêtes en Provence autrefois et aujourd'hui. Aix-en-Provence : Presses universitaires de Provence, 2014, p. 191.

STOUFF, Louis. Arles au Moyen-Âge finissant. Aix-en-Provence : Presses Universitaires de Provence, 2014.

STOUFF, Louis. « Arles angevine ». Dans ROUQUETTE, Jean-Maurice (dir.). Arles : histoire, territoires et cultures. Paris : Imprimerie nationale, 2008, p. 359.

TRUCHET, Michel de. « Mémoire sur les chevaux de Camargue ». Annales de l'agriculture française, t. XXVIII, 1807.

VENTURE, Remi. La confrérie des gardians et sa fête annuelle. Marguerittes : éditions Équinoxe, 1992. VENTURE, Remi. « Culture populaire arlésienne : de la tradition au folklore ». Dans ROUQUETTE, Jean-Maurice (dir.). Arles : histoire, territoires et cultures. Paris : Imprimerie nationale, 2008, p. 1041.

VENTURE, Remi. « La tauromachie à Arles : ébauche d'une histoire ». Dans ROUQUETTE, JeanMaurice (dir.). Arles : histoire, territoires et cultures. Paris : Imprimerie nationale, 2008, p. 1107.

\section{NOTES}

1. - Registre de la confrérie des gardians 1512-1539. Archives départementales des Bouches-duRhône 404 E 229. Registre de la confrérie des gardians 1634-1782. Médiathèque d'Arles Ms 293.

2. - Félibre : poète ou prosateur en langue d'Oc, membre du félibrige : école littéraire fondée en 1854.

3. - BERRIOT, Christian. Le cheval de Camargue. Lyon : imprimerie Bosc frères, 1969, p. 64.

4. - DURAND, Jacques. André Bouix, gardian de Camargue. Paris : Stock, 1980, p. 145.

5. - COULON, Louis. L'Ulysse françois ou le voyage de France, de Flandre et de Savoye. Paris : chez Gervais Clousier, 1643, p. 542.

6. - GALLON, Cécile. La confrérie Saint-Georges des gardiens d'Arles de 1634 à 1782. Nîmes : Sansouire, 2012, p. 20.

7. - NAUDOT, Carle. Camargue et gardians: ethnographie folklorique du pays d'Arles. Arles: Parc naturel régional de Camargue, 1989, p. 90-92.

8. - COULON, Louis, op. cit., p. 542.

9. - QUIQUERAN de BEAUJEU, Pierre. Louée soit la Provence (1551). Adaptation par Véronique Autheman. Arles : Actes Sud, 1999, p. 86-96.

10. - Ce principe a été observé de manière empirique par le maréchal-ferrant qui s'occupait des chevaux ibériques de l'écurie d'une célèbre rejoneadora installée en Camargue.

11. - QUIQUERAN de BEAUJEU, Pierre, op. cit., p. 102-104.

12. - BERRIOT, Christian, op. cit., p. 83-86.

13. - RUFUS, Jordanus. La science du cheval au Moyen-Âge, le traité d'hippiatrie de Jordanus Rufus. Éd. Brigitte Prévot. Paris : Klincksiek, 1991, p. 37-41.

14. - QUIQUERAN de BEAUJEU, Pierre, op. cit., p. 10. 


\section{RÉSUMÉS}

Le cheval de Camargue est étroitement lié au travail du gardian, pasteur cavalier chargé de la surveillance et de la conduite de troupeaux de bovins et de chevaux élevés en semi-liberté dans les zones incultes du delta du Rhône. Issu de traditions médiévales, ce métier est documenté à partir du XVI ${ }^{e}$ siècle et s'est perpétué dans des conditions et suivant des pratiques qui se sont maintenues jusqu'au début $\mathrm{du} \mathrm{XX}^{\mathrm{e}}$ siècle. Les spécificités de l'élevage des taureaux de Camargue ont conduit à la création de jeux taurins où le cheval, acteur incontournable, laisse modestement la vedette aux taureaux.

The 'gardians' of the Camargue and their horses, an archival and photographic record for a historical study. The Camargue horse is closely associated to the work of the 'gardians', mounted livestock guards in charge of keeping and driving the herds of bulls and horses raised in liberty in the uncultivated coastal part of the Camargue. Emerging from medieval traditions, this profession is well documented from the sixteenth century on and has been perpetuated up to the beginning of the twentieth century. The specific characteristic of bull breeding in the Camargue led to the use of young bulls to which the horse, an indispensable actor, modestly leaves pride of place.

\section{INDEX}

Keywords : Camargue, Arles, 'gardian', horse, bull, breeding, livestock, fraternity

Mots-clés : Camargue, Arles, gardians, cheval, taureau, élevage, troupeaux, confrérie

\section{AUTEUR}

\section{FRÉDÉRIC RAYNAUD}

chargé d'opérations et de recherches à l'INRAP, UMR 5648 frederic.raynaud@inrap.fr 